\title{
Role of Neuroepithelial Sonic hedgehog in Hypothalamic Patterning
}

\author{
Nora-Emöke Szabó, ${ }^{1}$ Tianyu Zhao, ${ }^{1}$ Murat Çankaya, ${ }^{2}$ Thomas Theil, ${ }^{3}$ Xunlei Zhou, ${ }^{1}$ and Gonzalo Alvarez-Bolado ${ }^{1,4}$ \\ ${ }^{1}$ Department of Genes and Behavior, Max Planck Institute of Biophysical Chemistry, D-37077 Göttingen, Germany, ${ }^{2}$ Department of Chemistry, Faculty of \\ Arts and Science, Atatürk University, 25240 Erzurum, Turkey, ${ }^{3}$ Centre for Neuroscience Research, University of Edinburgh, Edinburgh EH8 9XD, United \\ Kingdom, and ${ }^{4}$ Department of Tissue Engineering and Experimental Embryology, University of Tübingen, D-72074 Tübingen, Germany
}

The hypothalamus is a region of the diencephalon with particularly complex patterning. Sonic hedgehog (Shh), encoding a protein with key developmental roles, shows a peculiar and dynamic diencephalic expression pattern. Here, we use transgenic strategies and in vitro experiments to test the hypothesis that Shh expressed in the diencephalic neuroepithelium (neural Shh) coordinates tissue growth and patterning in the hypothalamus. Our results show that neural Shh coordinates anteroposterior and dorsoventral patterning in the hypothalamus and in the diencephalon-telencephalon junction. Neural Shh also coordinates mediolateral hypothalamic patterning, since it is necessary for the lateral hypothalamus to attain proper size and is required for the specification of hypocretin/orexin cells. Finally, neural Shh is necessary to maintain expression of differentiation markers including survival factor Foxb1.

\section{Introduction}

The hypothalamus is a ventral forebrain region regulating homeostasis and reproduction. Alterations of hypothalamic development can result in endocrine and metabolic disease (Michaud, 2001; Caqueret et al., 2005). Hypothalamic patterning, however, is not well understood. The embryonic hypothalamus forms the rostral-ventral part of the diencephalon. This is a complex region that, together with the caudal-dorsal diencephalon (prethalamic region), forms the diencephalon-telencephalon junction (DTJ). The adult hypothalamus consists of medial and lateral zones. The medial hypothalamus is a series of neuronal aggregates arranged rostrocaudally into four areas called preoptic, anterior, tuberal, and mammillary (MAM), each of them expressing specific markers. The lateral hypothalamus regulates ingestive, aggressive, and reproductive behaviors; without recognizable neuronal nuclei, it is anatomically and functionally very intricate (Simerly, 2004), and information about its specification and patterning is scarce. Specific lateral hypothalamus subpopulations expressing melanin-concentrating hormone (Pmch) and hypocretin ( $\mathrm{Hcrt}$ ) are essential for the coordination of sleep-wake cycles and feeding behavior (Burdakov et al., 2005).

Ventral patterning of the nervous system is controlled by signaling protein Shh, secreted by non-neural tissues like the prechordal plate and notochord (non-neural Shh) (Ericson et al., 1997; Gunhaga et al., 2000; Ingham and McMahon, 2001). However, Shh is also expressed by the ventral midline of the neural

\footnotetext{
Received March 5, 2009; revised April 21, 2009; accepted April 23, 2009.

This work was supported by The Max Planck Society. Dr. Ulrike Teichmann and her team took expert care of the mutant colonies.

This article is freely available online through the J Neurosci Open Choice option.

Correspondence should be addressed to Gonzalo Alvarez-Bolado, Department of Tissue Engineering and Experimental Embryology, Anatomisches Institut, Österbergstrasse 3, D-72074 Tübingen, Germany. E-mail: galvarez@anatom.uni-tuebingen.de.

DOI:10.1523/JNEUROSCI.1089-09.2009

Copyright $\odot 2009$ Society for Neuroscience $\quad$ 0270-6474/09/296989-14\$15.00/0
}

tube (neural Shh). Neural Shh is essential for the coordination of tissue growth and pattern in the midbrain-hindbrain junction and cerebellum (Blaess et al., 2006, 2008). In the diencephalic neuroepithelium, Shh shows an intriguing and dynamic pattern of expression with domains in the caudal-dorsal diencephalon [zona limitans interthalamica (ZLI)] and the rostral-ventral diencephalon (hypothalamic domain), which seem strategically situated to influence the formation of the DTJ. In the caudal diencephalon, neural Shh from the ZLI specifies the prethalamus (PTh) (Hashimoto-Torii et al., 2003; Kiecker and Lumsden, 2004; Vieira et al., 2005; Hirata et al., 2006; Scholpp et al., 2006; Guinazu et al., 2007) and promotes growth and differentiation of specific subdivisions of the thalamus (Szabó et al., 2009). The role of neural Shh in the rostral diencephalon (hypothalamus) and DTJ, however, is only starting to be analyzed.

We approached the role of neural Shh in hypothalamic development through analysis of a conditional mouse mutant as well as experiments in vitro. Our results show that neural Shh coordinates tissue growth with anteroposterior (AP) and dorsoventral (DV) patterning in the diencephalon and is essential for the formation of the DTJ. In the lateral hypothalamus, neural Shh is required for proper size of this zone and for the specification of hypocretin/orexin cells. Finally, neural Shh is necessary to maintain expression of differentiation markers including survival factor Foxb1.

\section{Materials and Methods}

Mutant mouse lines

Animals were treated in ways that minimize suffering and under authorization Az 32.22/Vo from the "Ordnungsamt der Stadt Göttingen," according to the German Law of Animal Protection.

Foxb1-Cre line. This line expresses Cre recombinase in the mouse diencephalon (Zhao et al., 2007, 2008), and it is a knock-in-knock-out generating Foxb1 heterozygous animals. These heterozygotes do not show haploinsufficiency and can be considered identical with wild type (WT) (Dou et al., 1997; Labosky et al., 1997; Alvarez-Bolado et al., 2000). 
Foxb1-Cre/ROSA26R lineage reporter line. In heterozygous Foxb1-Cre embryos carrying ROSA26 (Soriano, 1999) or Z/AP (Lobe et al., 1999) reporter alleles, all cells that express or have expressed Foxb1, and any cells derived from them, permanently produce $\beta$-galactosidase (ROSA26R) or human placental alkaline phosphatase (hPLAP; Z/AP), labeling the Foxb1 lineage (Zhao et al., 2007).

Conditional allele of Sonic hedgehog. In this conditional allele, exon 2 of Shh is flanked by loxP sites (Dassule et al., 2000; Lewis et al., 2001). Exon 2 encodes approximately one-half of the active N-terminal Shh signal, essential for Shh function (Mann and Beachy, 2004).

Shh-c mutant (Foxb1-Cre/Shh-fl conditional mutant). On crossing Foxb1-Cre and Shh-fl mice, Shh conditional mutant mice are produced. We analyze here only mice heterozygous for Foxb1-Cre and homozygous for Shh-floxed (Foxb1-Cre $e^{+/-}$Shh-fl ${ }^{-1-}$ ), which we term Shh-c mutants. No double homozygotes were used for the analysis.

In Shh-c mutants, exon 2 of the Shh locus has been deleted in the entire Foxb1 lineage, including the caudal diencephalon, the posterior ventral hypothalamus, and the diencephalic ventral midline (Zhao et al., 2007, 2008). In Shh-c mutants, whenever transcription from the Shh locus occurs (in cells of the Foxbl lineage), the recombined Shh locus produces a truncated, nonfunctional mRNA lacking exon 2. An exon 2 probe exclusively detects the functional $S h h$ mRNA (full length).

Shh full mutants. To generate full Shh mutants (see below) (see Fig. $1 I, J$ ), we crossed the $S h h$-floxed (Dassule et al., 2000) with a transgenic mouse line carrying Cre under an ubiquitous promoter expressed in embryonic stem cells (Schwenk et al., 1995), in this way producing full mutant homozygous embryos (CMV-Cre/Shh-floxed).

\section{Transfection constructs and electroporation}

To test the lack of function of $S h h$ without exon 2, we prepared constructs expressing exon 2-deleted Shh-IRES-EGFP [green fluorescent protein (GFP)] under the control of CAGGS promoter. We obtained a plasmid containing the full-length Shh cDNA (from imaGenes), clone IRAVp968F10144D, and we cloned this cDNA into pXL172 (CAGGS promoter-multicloning site-IRES-EGFP). Next, we used PCR to synthesize an exon 2-deleted Shh cDNA. In a first PCR step, we used Shh cDNA as template to amplify exon 1 and exon 3 in separate reactions. In each reaction, we used "bridge" primers so that the 3 ' primer for exon 1 contained $24 \mathrm{nt}$ of the $5^{\prime}$ end of exon 3 , and vice versa. We used the product of this amplification as template in a second PCR step, which produced a final continuous exon 1-exon 3 fragment (i.e., exon 2-deleted), which was also cloned into pXL172. The constructs were confirmed by sequencing.

Embryonic day 10.5 (E10.5) mouse brains were harvested, injected with $2 \mu \mathrm{g} / \mu \mathrm{l}$ DNA, and then electroporated with a CUY21EDIT Nepagene square wave electroporator (Nepa Gene) and the "tweezer electrodes" (22 V; 50 ms Pon, 950 ms Poff; three pulses). The brains were then cut open and cultured for $48 \mathrm{~h}$ (see below, Explants), and then GFP expression was analyzed and photographed, and the explants were fixed and treated for whole-mount in situ hybridization (ISH).

\section{ISH and reporter detection}

Templates were PCR-amplified (primer sequence available on request) from cDNA (from total RNA from newborn mouse brain plus E10.5E11.5 embryos). Probes were synthesized using the Roche RNA transcription kit (DIG RNA or Fluorescein RNA Labeling Mix). ISH on whole mount or on cryostat sections has been described often. For double whole-mount ISH, probes were labeled with either digoxygenin or fluorescein. Antibodies were as follows: anti-fluorescein-alkaline phosphatase (Fab fragment) (1:5000) and anti-digoxigenin-alkaline phosphatase (Fab fragment) (1:5000) (both from Roche). Color substrates were as follows: BM Purple, Fast Red, or INT (2-[4-iodophenyl]-3-[4nitrophenyl]-5-phenyltetrazolium chloride)/BCIP (5-bromo-4-chloro3-indolyl phosphate) (Roche).

Staining for alkaline phosphatase and for $\beta$-galactosidase activity were performed as described previously (Koenen et al., 1982; Lobe et al., 1999).

\section{5-Bromo-2'-deoxyuridine labeling}

Pregnant mice were intraperitoneally injected with 5-bromo-2'deoxyuridine (BrdU) (RPN201; GE Healthcare) (50 $\mu \mathrm{g} / \mathrm{g}$ of body weight) either at E10.5 or at E12.5, and the embryos were collected $1 \mathrm{~h}$ (E10.5) or $3 \mathrm{~h}$ (E12.5) later. We used anti-BrdU antibody M0744 (1:100) (Dako) on $20 \mu \mathrm{m}$ cryosections after epitope retrieval $(2 \mathrm{M} \mathrm{HCl}$ for $30 \mathrm{~min}$ at $37^{\circ} \mathrm{C}$ ), with nuclear marker $4^{\prime}, 6$-diamidino-2-phenylindole dihydrochloride (DAPI) (Invitrogen) as a counterstain. We examined and photographed the sections under confocal microscopy and counted BrdUlabeled and -unlabeled cells in the neuroepithelium on two histological sections (per level) at one rostral (anterior hypothalamic) and one caudal (MAM) level in three animals per age and genotype (wild type and Shhc). Then we calculated the labeling index (BrdU-labeled cells as percentage of total cells) (Takahashi et al., 1993; Warren et al., 1999; Ishibashi and McMahon, 2002).

\section{Apoptosis detection}

We selected cryostat sections of E10.5 and E12.5 brains at two hypothalamic rostrocaudal levels (anterior hypothalamic region and MAM) in three individuals per genotype and pretreated them with $4 \%$ paraformaldehyde (20 $\mathrm{min})$ and proteinase $\mathrm{K}(1.5 \mu \mathrm{g} / \mathrm{ml} ; 5 \mathrm{~min})$ at room temperature and then labeled the apoptotic cells with the ApopTag terminal deoxynucleotidyl transferase-mediated biotinylated UTP nick end labeling kit (Millipore) according to the instructions of the manufacturer. We counted absolute number of apoptotic cells per histological section of the hypothalamus (both sides) under high magnification with DAPI as counterstain.

\section{Statistical analysis}

ANOVA was performed with Prism software (GraphPad Software).

\section{Explants}

E9.5 wild-type mouse brains were cut open along the ventral midline, flattened (neuroepithelial side down) on Millicell-CM membranes (Millipore), and cultured under standard conditions in Neurobasal/glutamine (2 mM)/B-27 (Invitrogen). The following experimental reagents were added: Wnt pathway inhibitor 4-(4-(2,3-dihydrobenzo[1,4] dioxin6-yl)-5-pyridin-2-yl-1 H-imidazol-2-yl)benzamide (D4476) (Rena et al., 2004; Bryja et al., 2007) (50 $\mu \mathrm{m}$ in DMSO; Calbiochem), Shh pathway inhibitor cyclopamine (15 $\mu \mathrm{M}$ in DMSO; Sigma-Aldrich), and Wnt pathway activator $\mathrm{LiCl}$ (Klein and Melton, 1996) (10 mm; Sigma-Aldrich). Control experiments were performed in parallel with equal DMSO ( $\mathrm{NaCl}$ to control for $\mathrm{LiCl}$ ) concentrations. None of the reagents increased cell death ("Live/Dead" Viability/Cytotoxicity kit; Invitrogen) (data not shown). After $48 \mathrm{~h}$, explants were fixed (4\% paraformaldehyde) and processed for ISH. The Foxb1-expressing area of MAM neuroepithelium was measured on photographs with the help of CellF 2.6 software (Olympus Soft Imaging Solutions).

\section{Results}

\section{Foxb1-driven Cre abolishes Shh full-length expression in the anterior ventral neural plate and forebrain}

We crossed Foxb1-Cre heterozygous mice with Shh-floxed (Dassule et al., 2000) mice to obtain Foxb1-Cre ${ }^{+/-} /$Shh-floxed ${ }^{-1-}$ animals, termed Shh-conditional $(S h h-c)$ mutants. We did not use double homozygous animals for the analysis, because a Foxb1 $1^{-1-}$ phenotype could alter the Shh-c phenotype. Foxb $1^{+/-}$ are normal (see Materials and Methods). Shh-c mutants die around the time of birth. The patterning processes analyzed here take place much earlier.

The floxed Shh mouse line that we use has previously been used to analyze the role of $S h h$ in the development of other organs (Dassule et al., 2000; Lewis et al., 2001; Machold et al., 2003; Komada et al., 2008). On Cre-mediated recombination, the floxed Shh loses exon 2 (Dassule et al., 2000; Lewis et al., 2001), which encodes the $\mathrm{N}$ terminal, required for Shh function (Fan et al., 1995; Hynes et al., 1995; Lai et al., 1995; López-Martínez et al., 1995; Martí et al., 1995; Roelink et al., 1995). However, we wanted 
to ascertain that this was also the case in the forebrain neuroepithelium. E10.5 explants transfected with a construct expressing full-length Shh expressed ectopic Ptch1 (diagnostic of Shh pathway activation) (for review, see Lewis et al., 2001), whereas transfection of Shh constructs in which exon 2 had been deleted did not have any effect (Fig. $1 A-E$ ).

To analyze our mutants, we needed to know whether and when the mutant neural plate was able to express Shh. At E8.0, Foxb1 was expressed in the ventral midline of the wild-type anterior neural plate (Fig. $1 F$ ) (Zhao et al., 2007), whereas Shh was expressed in the prechordal mesendoderm but not yet in the neural plate of wild type or mutant (Fig. 1G,H). Foxb1 was expressed in the neural plate of a Shh ${ }^{-1-}$ embryo (full Shh knockout mutant), indicating that $S h h$ is not required for initiation of Foxb1 expression (Fig. $1 I, J$ ).

At E8.5, both Foxb1 and Shh were expressed in the wild-type ventral neural plate (Fig. $1 K, L$ ). In the mutant, the Shh locus was active as revealed by ISH with a "long probe" detecting any Shh transcripts (i.e., wild-type $S h h$ mRNA as well as truncated $S h h$ mRNA, the product of a Cre-recombined Shh locus) (Fig. $1 M$ ), but it produced nonfunctional Shh mRNA in the neural plate, as shown by an exon 2 probe, which detects only the product of the wild-type Shh locus (non-Cre-recombined) (see Materials and Methods) (Fig. 1N). At the 12-14 somite stage, Foxb1 and Shh were expressed in wild-type ventral diencephalon with a rostral limit at the optic sulcus (Fig. 1O,P, arrowhead) (Shimamura et al., 1995; Zhao et al., 2007). In the mutant, the Shh locus was active in appropriate domains (Fig. 1Q), but full-length, functional Shh transcripts were limited to the ventral caudal diencephalon (Fig. $1 R$, arrow), not rostral diencephalon (hypothalamus) (Fig. $1 R$, arrowhead).

These results indicated complete elimination of functional Shh mRNA in the Shh-c hypothalamus from E8.5 on.

\section{Activation of Shh expression in the basal plate depends on neural Shh}

Since diencephalic Foxb1 expression is mostly transient (Zhao et al., 2007, 2008), we used a lineage reporter mouse line to identify the regions in which a phenotype can be expected. In these embryos, cells that have expressed Foxb1, as well as cells derived from them, express $\beta$-galactosidase permanently, allowing for the identification of the regions in which Foxb1-driven Cre recombination has taken place (see Materials and Methods).

At 21-24 somites, Foxb1-lineage cells were found in the ventral part of rostral and caudal diencephalon, in the diencephalic ventral midline up to eye levels (Fig. $1 S$ ), and in the branchial arches (Fig. 1S, arrowheads). Shh at this age had disappeared from the ventral side (Fig. 1, compare $P, T$ ) and was expressed in four distinct domains: (1) suboptical, (2) a longitudinal band representing the basal plate (bp) of the rostral diencephalon (Puelles et al., 2004), (3) a small domain in the MAM, and (4) the incipient ZLI in the caudal diencephalon (Fig. 1T). The ventral portion of the tuberal area was devoid of Shh expression at this age (Fig. 1T, arrowhead). In Shh-c mutants, Shh transcriptional activation was abolished in the bp and ZLI domains, but maintained in the MAM and suboptical domains (Fig. $1 U$ ). Functional Shh was expressed only in the suboptical domain, with reduced intensity (Fig. $1 \mathrm{~V}$ ). In the ZLI domain, maintenance of activity in the Shh locus requires Shh (feedback loop) (Kiecker and Lumsden, 2004; Zeltser, 2005), which explains the lack of a ZLI in this mutant, and possibly also the lack of a bp domain. In contrast, the suboptical domain and the MAM domain can activate $S h h$ expression independently of Shh of neural origin.
The major brain regions can be recognized by expression of specific markers at E12.5 (Shimamura et al., 1995). At this age, the thalamus, the MAM, and scattered cells in prethalamus and hypothalamic floor plate were of Foxb1 lineage (Fig. $1 \mathrm{~W}$ ), indicating that those regions would not be able to express functional $S h h$ in the mutant. In the wild-type embryo at this age, a new Shh domain was present [medial ganglionic eminence (MGE)] and the domains detected earlier were fully developed (Fig. $1 X$ ). The MAM showed an Emx2-expressing subdomain corresponding to its most prominent neuronal nucleus, the mammillary body (MBO) (Fig. $1 X$, inset; supplemental Fig. $1 A$, available at www. jneurosci.org as supplemental material) and a Shh-expressing subdomain. The bp domain of Shh expression was still strong in the tuberal area (Fig. $1 X$ ). In the $S h h-c$ brain, the bp domain was absent (as well as the ZLI) (Fig. 1Y), and most of the remnant Shh transcriptional activity produced inactive $S h h$ transcripts, except for the MGE (Fig. 1Z).

\section{Early abolition of the $S h h$ pathway in the $S h h-c$ forebrain}

Non-neural sources of Shh can still activate the Shh pathway in the mutant neuroepithelium. Therefore, we characterized the state of the pathway in Shh-c embryos by detecting expression of Shh receptor Ptch1 and transcription factor gene Gli1, diagnostic markers of Shh pathway activity (for review, see Lewis et al., 2001). At E8.5, the pathway was active in the mutant ventral midline (Fig. $2 A-D$ ), probably because of a response to nonneural Shh from the prechordal plate. At 14 somites, Gli1 and Ptch 1 expression in the mutant disappeared from the ventral diencephalon (Fig. $2 E-H$ ) but was retained in the ventral telencephalon further demonstrating the specific ablation of Shh in the diencephalon (Fig. $2 H$ ). The loss was maintained at 24 somites (Fig. $2 I-L$ ). These results show a shift in the dependence of the wild-type neural plate from non-neural to neural sources of Shh and indicate that, in the Shh-c neural plate, the Shh pathway is rendered inactive very early at diencephalic levels.

\section{Regional hypothalamic patterning in the Shh-c embryo}

Shh is essential to specify the hypothalamus (Chiang et al., 1996). Regional specification of the hypothalamus is defined by $N k \times 2-1$ (Titf1) expression (Kimura et al., 1996; Puelles et al., 2004). We found $N k \times 2-1$ expression in an appropriate (although reduced in size) region of the Shh-c forebrain at E9.5 (Fig. $3 A, B$ ) and E11.5 (Fig. $3 C, D$ ), indicating the presence of a ventral forebrain region fated to become hypothalamus.

The major brain regions can be recognized by specific marker expression at E12.5 (Shimamura et al., 1995). To explore the regionalization of the hypothalamus in the absence of neural Shh, we detected expression of $D l \times 2$ (Pierani et al., 2001; Petryniak et al., 2007) and Dbx1 (Lu et al., 1992; Shoji et al., 1996), transcription factor genes expressed in the presumptive hypothalamus and with important developmental roles. ( $N k \times 2-1$ at E12.5 can be seen in Fig. $4 A$, B.) Expression of $D l x 2$ (Fig. $3 E, F$ ) and $D b x 1$ (Fig. $3 G, H)$ was almost completely abolished in the mutant hypothalamus.

\section{The MAM in the Shh-c embryo}

Dlx2 expression in the mutant (Fig. $3 F$ ) suggests abolition of the PTh. This could be expected, since neural Shh (from the ZLI) is essential for PTh development, as has been thoroughly documented (see references in Introduction). However, the continuity of dorsal and ventral diencephalon in the absence of a PTh has not been investigated. Rostral to the ZLI, the diencephalon consists of PTh and thalamic eminence [eminentia thalamica 

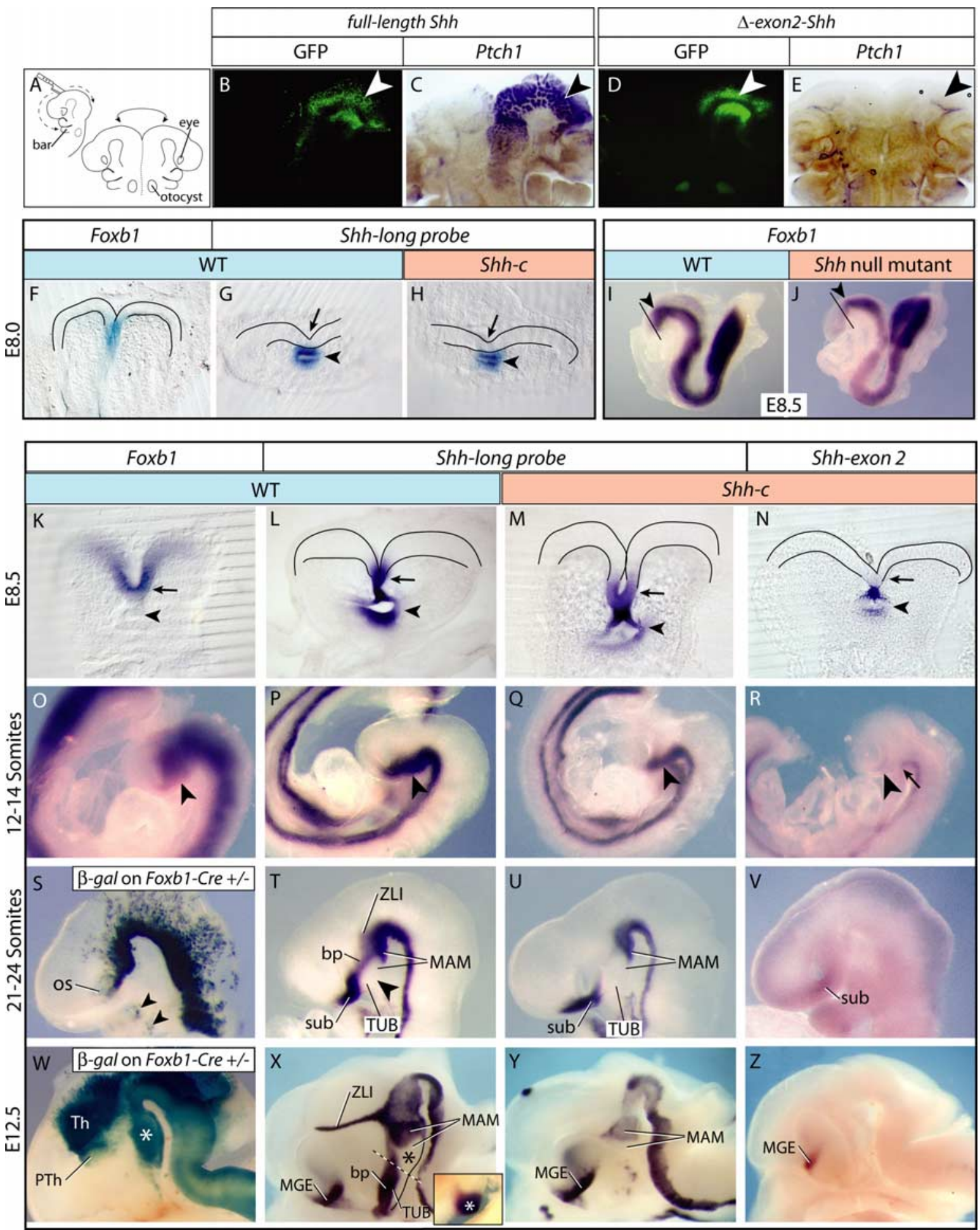

Figure 1. Foxb1-driven Cre abolishes Shh expression in the anterior ventral neural plate and forebrain. $\boldsymbol{A}-\boldsymbol{E}$, Explant cultures according to $\boldsymbol{A}$ of E10.5 wild-type brains ( $\boldsymbol{B}-\boldsymbol{E})$ electroporated with full-length

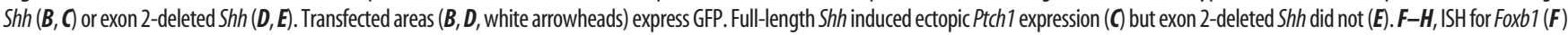
and Shh-long probe $(\boldsymbol{G}, \boldsymbol{H})$ on E8.0 anterior neural plate in wild type $(\boldsymbol{F}, \boldsymbol{G})$ and $\operatorname{Shh}$ - $(\boldsymbol{H})$. The ventral neural plate shows Foxb1 $(\boldsymbol{F})$, but not yet $\operatorname{Shh}$ in wild type $(\boldsymbol{G})$ or mutant $(\boldsymbol{H}) . \boldsymbol{I}, \boldsymbol{J}$, Foxb1 is expressed in the early neural plate in wild type $(\boldsymbol{I})$ and $S h^{-I-}$ (full mutant) $(\boldsymbol{J})$. The arrowhead indicates the diencephalon. The black line indicates the DTJ. $\boldsymbol{K}-\boldsymbol{N}$, At E8.5, Foxb1 and Shh are coexpressed in wild-type ventral anterior neural plate $(\boldsymbol{K}, \boldsymbol{L}$, arrow). Shh is expressed in foregut also $(\boldsymbol{K}, \boldsymbol{L}$, arrowhead). In the mutant, Shh is expressed in the ventral rostral neural plate $(\boldsymbol{M}$, arrow) and foregut $(\boldsymbol{M}$, arrowhead) but lacks exon 2 in

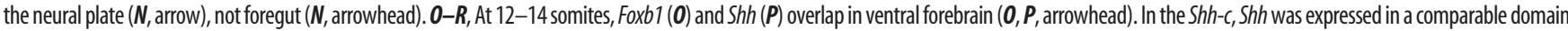
$(\boldsymbol{Q}$, arrowhead), but lacked exon 2 ( $\boldsymbol{R}$, arrowhead). The tegmentum expressed some full-length $\operatorname{Sh}(\boldsymbol{R}$, arrow). $\boldsymbol{S}-\boldsymbol{V}, \boldsymbol{\beta}$-Galactosidase shows Foxb 1 lineage in Foxb1-Cre/ROSA26R mice at $21-24$ somites ( $\boldsymbol{S})$ overlapping with Shh expression ( $\boldsymbol{T}$ ) (arrowheads in Sshow Foxb7-lineage cells in the branchial arches). Shh expression ( $\boldsymbol{T}$ ) was absentfrom ventral forebrain (arrowhead) and showed novel domains: suboptical, basal plate (bp), ZLl, and MAM. In the mutant, Shh transcriptional activation was missing in the bp and ZLI domains (U). Functional Shh transcripts were produced only in the sub, in reduced amounts (V). $\boldsymbol{W}-\boldsymbol{Z}$ At E12.5 the Foxb1 lineage covers thalamus and most of the prethalamus (Th and PTh in $\boldsymbol{W}$ ), as well as MAM ( $\boldsymbol{W}$, asterisk). Shh expression ( $\boldsymbol{X}$ ) showed a telencephalic domain (MGE). Dotted line, TUB-MAM boundary. In the MAM, the MBO ( $\boldsymbol{X}$, asterisk) does not express Shh but Emx2 ( $\boldsymbol{X}$, inset) (see also supplemental Fig. $1 A$, available at www.jneurosci.org as supplemental material). In the mutant $(\boldsymbol{Y})$, only the MAM and MGE domains remained. Only the MGE produced functional Shh transcripts (Z). For the abbreviations used in the figures, see Table 1. 


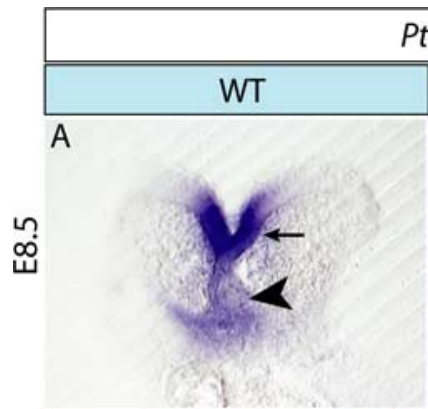

Ptch1 Shh-c
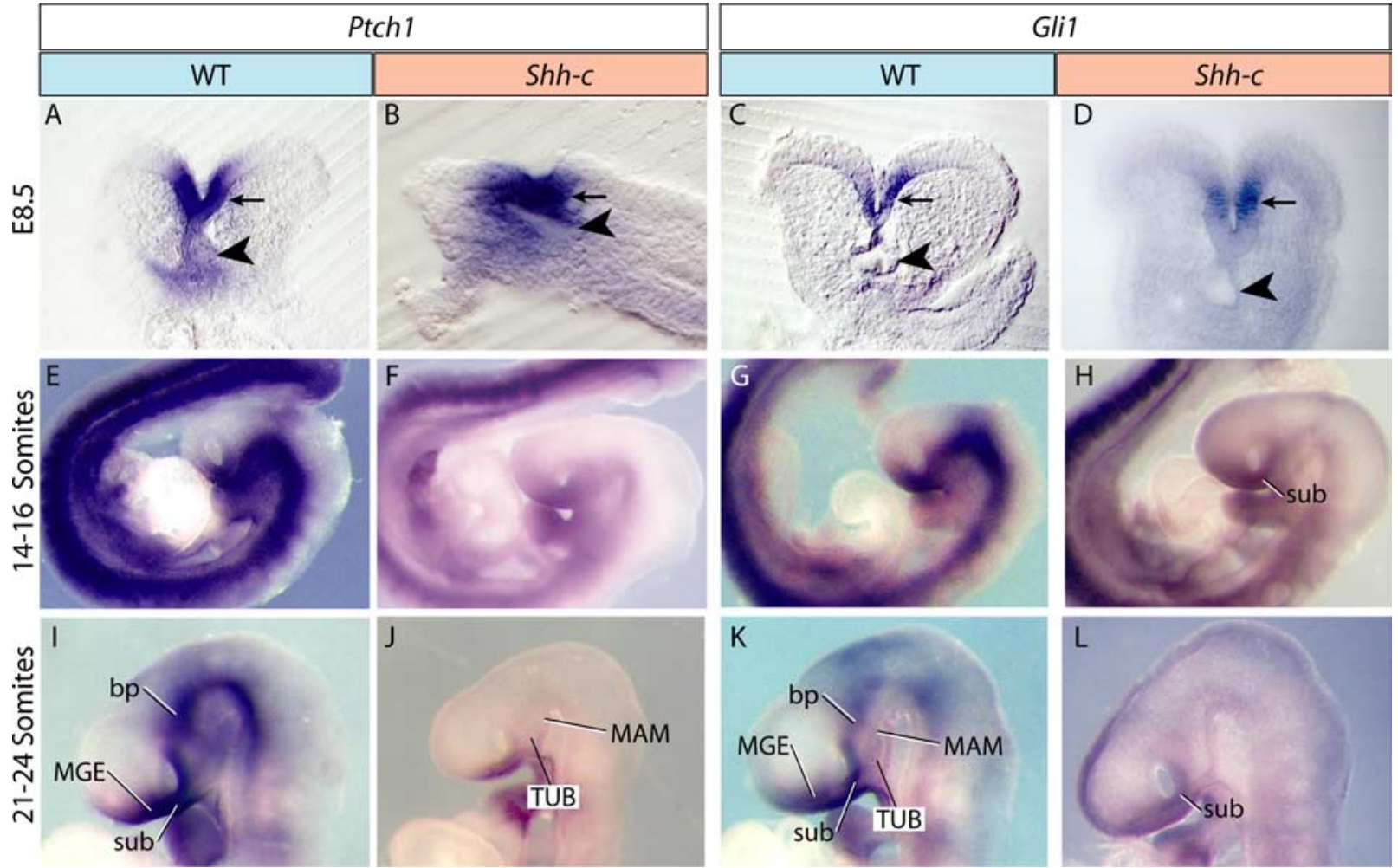

Figure 2. The Shh pathway is gradually abolished in the early Shh-c forebrain. In situ hybridization for Ptch1 and Gli1 on wild-type and Shh-c mutant embryos. A-D, At E8.5, Ptch1 and Gli1 were expressed in the ventral rostral neural plate (arrow) in wild type $(\boldsymbol{A}, \boldsymbol{C})$ and mutant $(\boldsymbol{B}, \boldsymbol{D})$. The arrowheads indicate the foregut. $\boldsymbol{E}-\boldsymbol{H}$, At $14-16$ somites, both Ptch1 and $G$ li1 were expressed in the entire ventral neural tube $(\boldsymbol{E}, \boldsymbol{G})$ but had disappeared from the mutant rostral neural tube $(\boldsymbol{F}, \boldsymbol{H})$ except in the sub. $\boldsymbol{I}-\boldsymbol{L}$, At $21-24$ somites, the domain of expression of Ptch1 and $G$ li1 in wild type $(\boldsymbol{I}$, $\boldsymbol{K}$ ) showed domains similar to Shh: bp and sub, and an incipient telencephalic domain, MGE (compare with Fig. $1 T$ ), whereas in the mutant both markers were still absent from the forebrain $(\boldsymbol{J}, \mathbf{L}$ ) except for low-intensity expression of Gli1 in the sub $(\boldsymbol{L})$.

(EMT) ]. Lhx5 labels specifically the EMT and the MBO, but not the PTh (Bachy et al., 2001) (Fig. 3I). In the mutant, an abnormal $L h x 5$ domain encompassed a stripe of dorsal and ventral diencephalon (Fig. 3J), as if formed by a fusion between EMT and MAM. Colocalization of this marker with Pitx2, a specific marker of the MAM (Skidmore et al., 2007), showed that the MAM is not dorsally expanded in the mutant, but the absence of PTh tissue leads to abnormal contiguity of EMT and MAM (Fig. $3 K, L$ ).

Wnt8b expression labels the $\mathrm{MBO}$ in all vertebrates (Cui et al., 1995; Kelly et al., 1995; Lako et al., 1998; Garda et al., 2002; Houart et al., 2002), as well as the rostral border of the dorsal diencephalon (supplemental Fig. $1 B$, available at www.jneurosci. org as supplemental material). In the Shh-c mutant, both domains coalesce (supplemental Fig. $1 C$, available at www.jneurosci.org as supplemental material) like the $L h x 5$ domains (Fig. $3 J$ ). Codetection of Wnt $8 b$ with Pitx2 (supplemental Fig. 1D,E, available at www. jneurosci.org as supplemental material) confirmed that both domains become contiguous in the mutant, as opposed to a dorsal expansion of the MAM. The fact that $L h \times 5$ is expressed in a mutant without PTh represents a genetic dissection of the rostral-dorsal diencephalon. We analyzed this by colocalizing EMT marker Tbr1 (Puelles et al., 2000) and PTh marker Arx (Kitamura et al., 1997), which show contiguous domains in the rostral-dorsal diencephalon (supplemental Fig. 1F, available at www. jneurosci.org as supplemental material). As expected, Arx was missing in the mutant, but Tbrl also (supplemental Fig. $1 G$, available at www.jneurosci.org as supplemental material). [The wild-type expression patterns of Foxg1, Foxd1, Tbr1, and Pitx2 as single markers can be found in supplemental Fig. $1 H-K$ (available at www.jneurosci.org as supplemental material).]

The apparent fusion of rostral-dorsal diencephalon with the MAM results in an alteration of the DTJ. The expression domains of transcription factor genes Foxd1 and Foxg1 define the DTJ (Hatini et al., 1994; Herrera et al., 2004) (Fig. 3M). Codetection of these markers in the mutant was less informative, since Foxd1 failed to be expressed (Fig. $3 N$ ). However, careful examination of the DTJ in our material showed that, in the mutant, it goes all the way to the ventral side, as shown in the diagrams in Figure 3, $O$ and $P$.

\section{The DTJ in the Shh-c}

To examine the DTJ more closely, we sectioned E12.5 wild-type and mutant embryos along the horizontal plane of section (indicated in Fig. $4 A, B, K, L)$ and labeled them for hypothalamic marker Nkx2-1 as well as MBO marker Emx2 (Fig. $4 A, B, K, L$ ). $N k \times 2-1$ labeling showed that the mutant tuberal area was much shorter along the anteroposterior axis (Fig. $4 \mathrm{E}-\mathrm{H}$ ) and had lost $N k \times 2-1$ expression in a large caudal area (Fig. $4 C-J$ ).

The most dorsal expression of $N k \times 2-1$ in the hypothalamus (Fig. $4 A$, arrow) marked a depression or sulcus in the neural tube (Fig. 4C, arrow), which could be followed to ventral levels in the wild type (Fig. $4 C, E, G$, arrows) and the mutant (Fig. $4 D, F, H$, J, arrows).

Emx2 expression labeled the $\mathrm{MBO}$ very specifically in the wild type (Fig. $4 \mathrm{~K}, \mathrm{~S}$; supplemental Fig. $1 \mathrm{~A}$, available at www. jneurosci.org as supplemental material), and in the mutant it was present in an abnormal caudal structure (Fig. $4 L$, arrowhead). Emx2-labeled sections of the mutant showed that the sulcus mentioned above starts at the point (Fig. $4 \mathrm{~N}$, arrow) at which the 
Table 1. Abbreviations

\begin{tabular}{ll}
\hline Abbreviation & Definition \\
\hline 3V & Third ventricle \\
ac & Anterior commissure \\
AHA & Anterior hypothalamic area \\
bar & Branchial arch \\
bp & Basal plate \\
CTX & Cortex \\
DG & Dentate gyrus \\
DTJ & Diencephalon-telencephalon junction \\
EMT & Thalamic eminence (eminentia thalamica) \\
hPLAP & Human placental alkaline phosphatase \\
Hy & Hypothalamus \\
LH & Lateral hypothalamus \\
MAM & Mammillary area \\
MBO & Mammillary body \\
MGE & Medial ganglionic eminence \\
OS & Optic sulcus \\
Oto & Otocyst \\
PCP & Prechordal plate \\
PRO & Preoptic area \\
PT & Pretectum \\
PTh & Prethalamus \\
PVN & Paraventricular nucleus \\
SC & Superior colliculus \\
SCH & Suprachiasmatic nucleus \\
Sub & Suboptical domain \\
TG & Tegmentum \\
Th & Thalamus \\
TL & Telencephalon \\
TUB & Tuberal area \\
VL & Lateral ventricle \\
VMH & Ventromedial nucleus \\
ZLI & \\
\hline & \\
& Zonitans interthalamica \\
\hline
\end{tabular}

cortex (telencephalon) meets the diencephalon, in coincidence with the beginning of an Emx2-expressing hypothalamic region which seems an extension of the cortex (Fig. $4 N$, arrowhead). The sulcus could be followed in the ventral direction in the wild type (Fig. $4 M, O, Q$ ) and mutant (Fig. $4 N, P, R, T$ ).

However, in the Shh-c hypothalamus, Emx2 expression labeled a region flattened rostrocaudally and with a convoluted lumen (Fig. $4 N, P, R, T$, arrow and arrowhead) more reminiscent of cortex than hypothalamus.

\section{An Emx1-expressing layered structure in the ventral diencephalon of the Shh-c embryo}

$E m \times 1$ is a very specific cortical marker not expressed in any other structure (Gulisano et al., 1996) and labels the entire cortex of the E12.5 mouse (Fig. $5 A, B$ ). In the Shh-c mutant, intriguingly, the Emx1-expressing cortex extended toward the midline (Fig. 5C,D, red arrowheads). In coronal sections (Fig. $5 E-H$ ) of wild type and mutant, we could ascertain expression of Emx1 in one layered structure occupying the ventral side of the diencephalon (Fig. $5 G, H$, red arrowheads). Detection of $E m x 1$ at E18.5 (Fig. 5J,K) confirmed this finding (Fig. $5 K$, red arrowheads). Tbr1 is a marker of the pallium (cortex and hippocampus) and thalamic eminence (Bulfone et al., 1995) and it was expressed in a layered structure positioned in the mutant hypothalamus at E18.5 (Fig. $5 L, M$ ). Foxg1 (Xuan et al., 1995) is a telencephalic marker (Fig. $5 N$ ) also expressed in this structure (Fig. 5O, arrow).

\section{Cell proliferation and cell death in the Shh-c hypothalamic neuroepithelium at E10.5 and E12.5}

Shh promotes proliferation and cell viability in the diencephalic neuroepithelium at E9.0 (Ishibashi and McMahon, 2002). We wanted to know whether neural Shh controls those cellular processes in the hypothalamus after that age. To address this question, we analyzed $S h h-c$ mutant embryos for proliferation and cell death. We analyzed proliferation by BrdU labeling at E10.5 (1 h survival) and E12.5 (3 h survival). At E10.5, we did not find any difference in labeling index between wild type and mutant (data not shown). At E12.5, however, we found in rostral sections a moderate $(\sim 20 \%)$ but significant $(p<0.001)$ decrease in proliferation in the mutant (labeling index in rostral hypothalamus: $49.5 \pm 0.70$ in wild type vs $40 \pm 4.20$ in the mutant; in caudal hypothalamus: $50 \pm 0.5$ in wild type vs $51.5 \pm 0.70$ in the mutant) (data not shown). We did not find changes in cell death in the hypothalamus at E10.5 or E12.5 (data not shown).

\section{The ventral diencephalon of the Shh-c is transversally divided into two parts}

Next, we wanted to assess diencephalic differentiation at a later stage, when neurogenesis is over and the mantle layer is differentiated. Since Shh-c mutants do not survive beyond the end of gestation, we analyzed E18.5 brains. Inspection of Nissl-stained sections showed size reduction of the basal ganglia, hypothalamus and thalamic region, and an enlarged third ventricle (Fig. $6 A, B)$. As expected from previous results (Fig. $5 K$ ), a layered structure (Fig. $6 B$, asterisk) was positioned medially and ventrally. To identify the major brain regions in the mutant, we used marker genes Gbx2, Nkx2-1, Lhx1, and Calb1. Gbx2, a thalamic transcription factor (Miyashita-Lin et al., 1999), identified a reduced mutant thalamus (Fig. 6C,D). Expression of $N k \times 2-1$, an essential marker of the early hypothalamus as a region, is restricted during differentiation to specific hypothalamic nuclei including part of the preoptic area and the mamillary body (Price et al., 1992) (Fig. 6E). Expression of $N k x 2-1$ was maintained in the mutant preoptic area (Fig. $6 F$ ). Immediately caudal to the preoptic area is the anterior hypothalamic area, a prominent nucleus of which is the suprachiasmatic ( $\mathrm{SCH})$, specifically expressing transcription factor $L h x 1$. Lhxl expression was preserved in the mutant (Fig. $6 G, H$ ). The next rostrocaudal subdivision, the tuberal region, contains the ventromedial nucleus (VMH), specifically expressing Calb1 (Fig. 6I). In the mutant, the VMH was smaller but expressed the marker (Fig. $6 J$ ). The layered structure was positioned immediately caudal to the VMH in the mutant (Fig. $6 J$ ) but rostral to the MBO (Fig. 6F,H). This suggested that this structure separated the preoptic, anterior, and tuberal areas from the MAM.

These results are summarized in Figure 6, $K$ and $L$. Additional information can be found in supplemental Figure 2 (available at www.jneurosci.org as supplemental material).

\section{Neural Shh is required for the development of the lateral hypothalamus}

To explore the mediolateral extension of the mutant hypothalamus we detected expression of arginine vasopressin ( $A v p)$, which encodes a hormone specifically expressed in the paraventricular (PVN) and supraoptic (SO) nuclei (Fig. 7A). In the mutant, the PVN was reduced, $A v p$ neurons were scattered in the mediolateral plane and the SO was absent (Fig. $7 B$ ). However, on sagittal sections labeled with specific marker genes Sim1 (Michaud et al., 1998) and Otp (Acampora et al., 1999) (supplemental Fig. 2 E-H, available at www.jneurosci.org as supplemental material), the PVN was not expanded rostrocaudally. Since the hypothalamus is strongly patterned in the transverse plane (i.e., medial hypothalamus vs lateral hypothalamus), these data suggested a specific alteration of mediolateral patterning.

In horizontal sections, the lateral hypothalamus can be recog- 

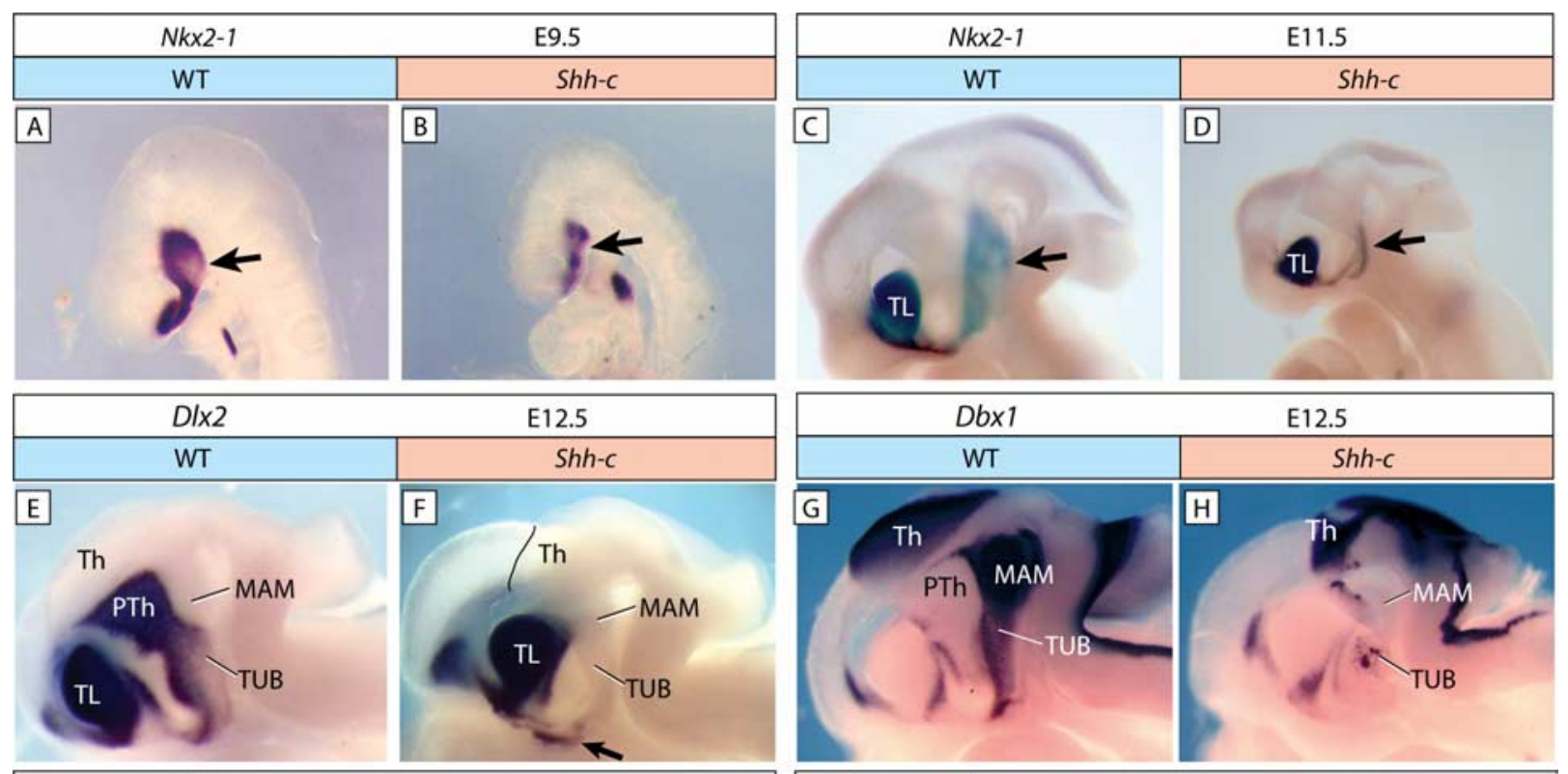

\begin{tabular}{|c|c|}
\hline Lhx5 & E12.5 \\
\hline WT & Shh-c \\
\hline \hline
\end{tabular}
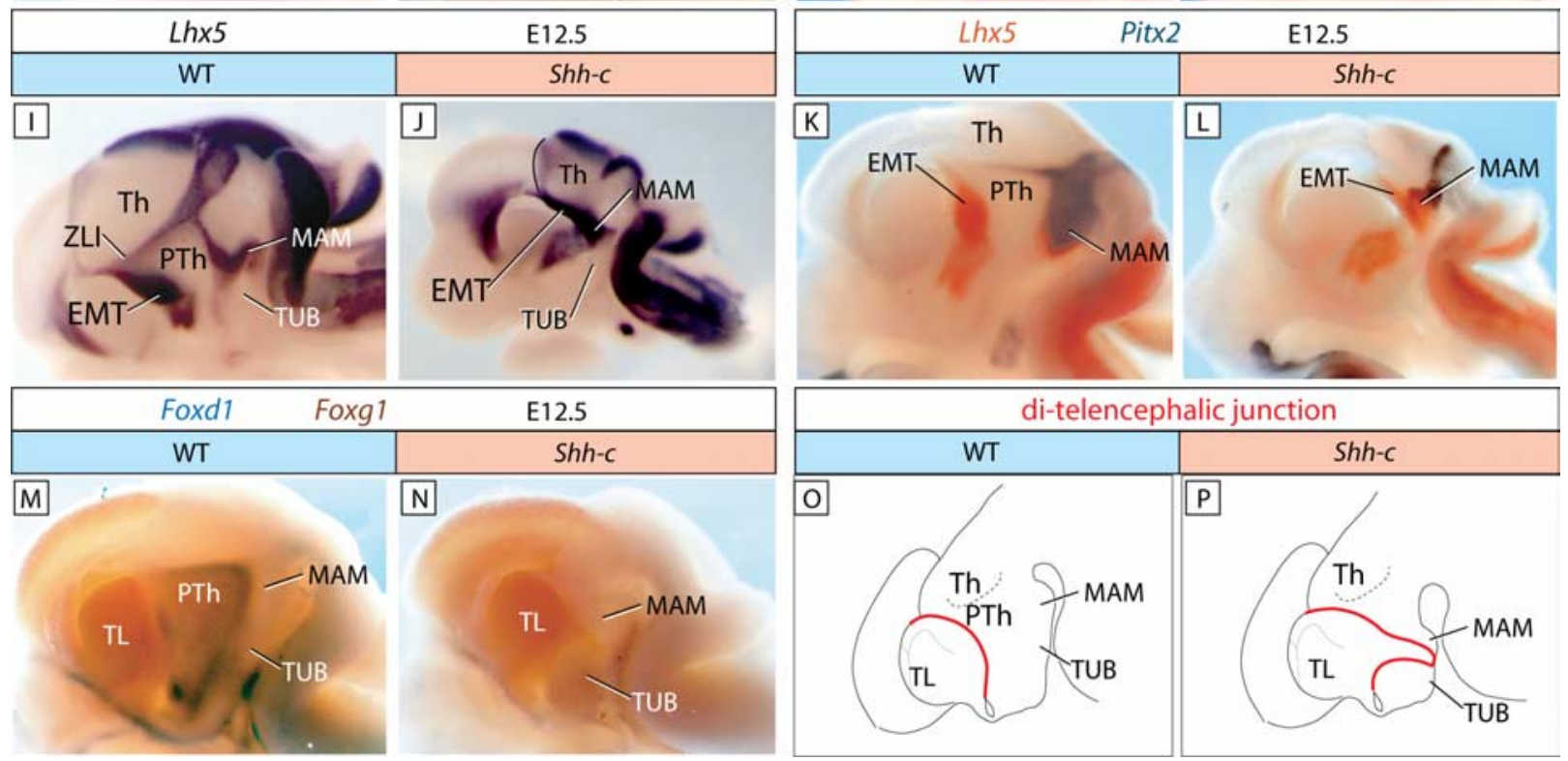

Figure 3. Loss of hypothalamic markers in the Shh-cembryo. $\boldsymbol{A}-\boldsymbol{N}$, Whole-mount ISH detection on hemisected embryo brains. Ages, probes, and genotypes are as indicated. $\boldsymbol{A}-\boldsymbol{D}, \mathrm{At}$ E9.5 $(\boldsymbol{A}, \boldsymbol{B})$ as well as E11.5 $(\boldsymbol{C}, \boldsymbol{D}), N k \times 2-1$ was expressed in the ventral forebrain in wild type $(\boldsymbol{A}, \boldsymbol{C}$, arrows) and mutant $(\boldsymbol{B}, \boldsymbol{D}$, arrows). $\boldsymbol{E}, \boldsymbol{F}$, The TUB (and PTh) domains of $D / \times 2(\boldsymbol{E})$ were almost completely abolished in the mutant $(\boldsymbol{F})$ (the arrow indicates a remnant). The upper border of Th is outlined for clarity. $\boldsymbol{G}, \boldsymbol{H}$, The hypothalamic domain of $D b x 1$ expression (G) (MAM plus TUB) was absent in the mutant $(\boldsymbol{H}) . \boldsymbol{I}, \boldsymbol{J}$, The MAM and EMT domains of $L$ hx $5(\boldsymbol{I})$ are reduced and seem fused in the mutant $(\boldsymbol{J}) . \boldsymbol{K}, \boldsymbol{L}$, Colocalization of $L h \times 5$ and Pitxz expression clarifies the extent and position of the MAM with respect to the EMT in the mutant. $\boldsymbol{M}, \boldsymbol{N}$, Foxg1 and Foxd 1 show the DTJ in wild type $(\boldsymbol{M})$. In the mutant, Foxd 1 is not expressed $(\boldsymbol{N}) . \mathbf{0}, \boldsymbol{P}$, Diagrams showing the DTJ (red line) in wild-type (0) and Shh-C (P) E12.5 hemisected brains as seen in the previous panels.

nized by the large number of longitudinal axons that course through it, as opposed to the more compact medial hypothalamus (Fig. 7C). By this criterion, a lateral hypothalamus seems to be mostly absent in the mutant (Fig. 7D). By crossing our Shh-c conditional mutants with the mouse reporter line Z/AP (Lobe et al., 1999), we labeled the neurons of Foxb1 lineage (see Materials and Methods), which are very abundant in the lateral hypothalamus (Fig. 7E) but were dramatically reduced in the mutant (Fig. $7 F)$. Specific expression of marker genes pro-melaninconcentrating hormone (Pmch) (Fig. 7G) and hypocretin-orexin (Hcrt) (Fig. 7I) characterizes two independent neuronal subpopulations in the lateral hypothalamus. The number of Pmch neurons was extremely reduced in the mutant (Fig. $7 H$ ), whereas Hcrt neurons were completely absent (Fig. $7 J$ ). These results (summarized in Fig. $7 K, L$ ) show that deficiency in neural Shh results in alterations of growth and terminal differentiation of the lateral hypothalamus as well as in the lack of specific, functionally important neuronal subpopulations.

\section{Neural $S h h$ is required to maintain expression of the mammillary survival factor Foxb 1}

Dependence of Foxb1 expression on neural Shh (Fig. 8A,B) was surprising, since Foxbl is expressed in the Shh-deficient neural plate (Fig. $1 I, J$ ), suggesting a feedback loop between both factors.

We analyzed this question on cultured explants of whole embryonic forebrain. For this purpose, we dissected the forebrains of wild-type E9.5 mouse embryos, cut them open following the ventral midline, and flattened them on Millipore membranes 

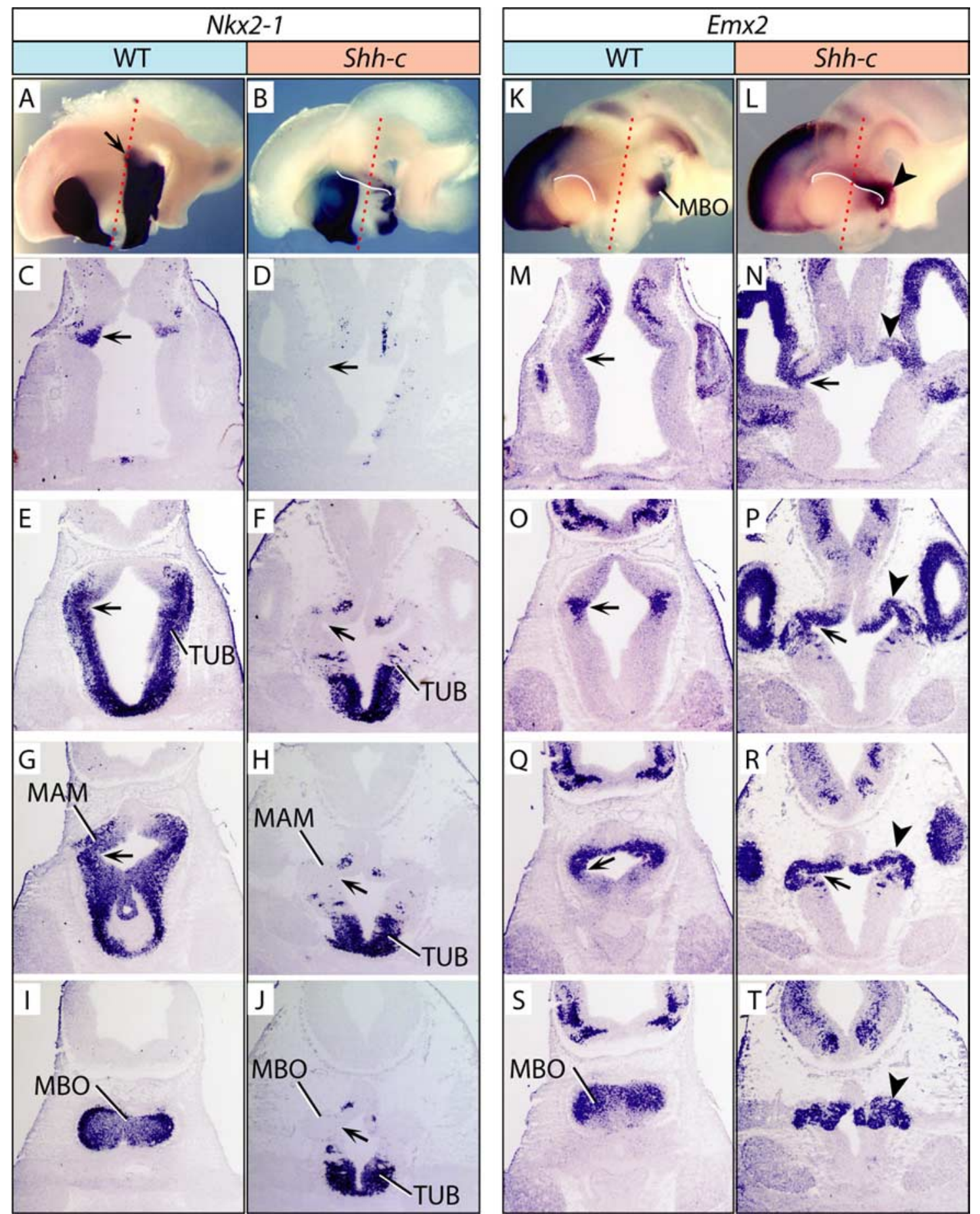

Figure 4. The Shh-c hypothalamus in horizontal sections at E12.5. $A, B, K, L$, Whole-mount detection of gene expression on hemisected E12.5 brains; probes and genotypes are as indicated. The red dotted lines indicate approximate plane of section of the panels below. The white lines in $\boldsymbol{B}, \boldsymbol{K}$, and $\boldsymbol{L}$ emphasize the DTJ. $\mathbf{C}-\boldsymbol{J}, \boldsymbol{M}-\boldsymbol{T}$, ISH detection of $N k \times 2-1(\boldsymbol{C}-\boldsymbol{J})$, and $E m \times 2(\boldsymbol{M}-\boldsymbol{T})$ on horizontal sections showing four dorsal-to-ventral levels of the hypothalamus of WT and Shh-C E12.5 brains as indicated. The most dorsal hypothalamic domain of $N k \times 2-1$ ( $A$, $C$, arrows) in the wild type marks a sulcus that can be followed up to ventral levels in wild type and mutant $(\boldsymbol{D}-\boldsymbol{H}, \boldsymbol{J}, \boldsymbol{M - R}$, arrows). $\boldsymbol{M}, \boldsymbol{N}$, Emx2 expression shows that the sulcus corresponds in the mutant to the point at which diencephalon and telencephalon meet ( $\boldsymbol{N}$, arrow), in close proximity to a domain of $E m \times 2$ expression ( $\boldsymbol{N}$, arrowhead). This $E m \times 2$ domain can be followed in the mutant from the DTJ ( $\boldsymbol{N})$ to ventral levels of the hypothalamus $(\boldsymbol{P}, \boldsymbol{R}, \boldsymbol{T})$. S, Expression of Emx2 in the wild-type MBO. $\boldsymbol{T}$, A convoluted structure expresses Emx2 in the mutant.

with the neuroepithelial side down (Fig. 8C-E). The explants were cultured for $48 \mathrm{~h}$ in control media or after adding one of several reagents to modify the activity of different signaling pathways. Shh pathway inhibitor cyclopamine abolished Foxb1 expression in E9.5 explants (in 8 of 10 explants; 8 of 10) (Fig. $8 F, G$ ), but not in E12.5 explants (9 of 11) (Fig. 8 H,I). Therefore, between E9.5 and E12.5, neural Shh maintains Foxb1 expression, perhaps against inhibiting influences by other factors. A powerful signaling agent, Wnt8b, specifically expressed in the MBO (Fig. $8 J-L)$ is able to reduce the Foxb1 MAM expression domain in zebrafish (Kim et al., 2002). We therefore tested whether canonical Wnt signaling controls Foxb1 expression. Wnt pathway activator $\mathrm{LiCl}$ (Stambolic et al., 1996) decreased the Foxb1 MAM expression domain (9 of 10) (Fig. 8M,N). D4476 is a casein 


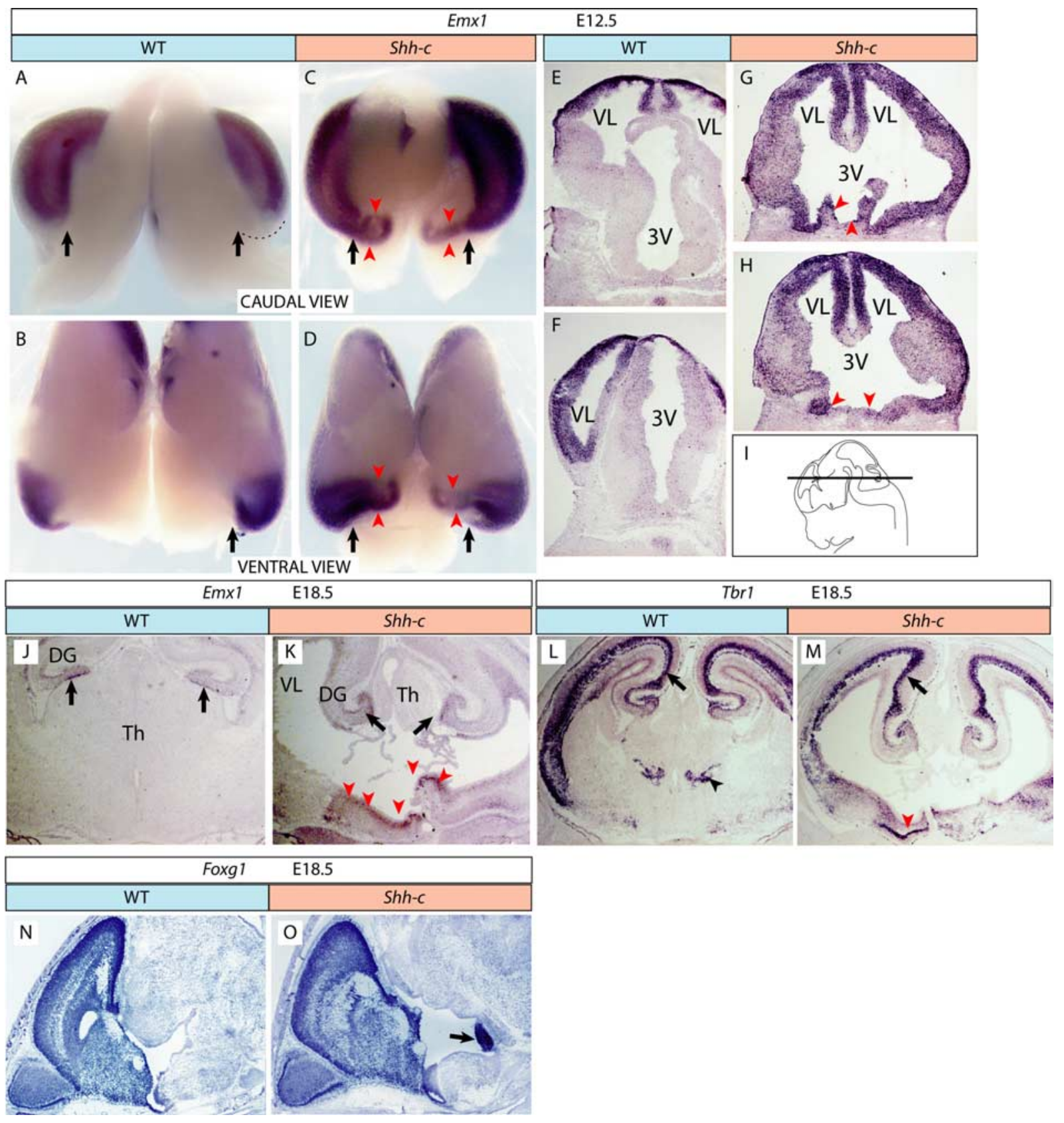

Figure 5. Layered structure in the ventral diencephalon in the Shh-c brain. $\boldsymbol{A}-\boldsymbol{D}, \mathrm{E} 12.5$ wild-type $(\boldsymbol{A}, \boldsymbol{B})$ and $\operatorname{Shh}-\boldsymbol{C}(\boldsymbol{C}, \boldsymbol{D})$ brains hybridized for $E m \times 1$ in caudal $(\boldsymbol{A}, \boldsymbol{C})$ and ventral $(\boldsymbol{B}, \boldsymbol{D})$ view (the diencephalic ventral side was cut open longitudinally). The black arrows indicate the medial limit of the cortex in wild type $(\boldsymbol{A}, \boldsymbol{B})$ and comparable point in the mutant $(\boldsymbol{C}, \boldsymbol{D})$. The red arrowheads indicate ectopic expression of Emx 1 in the mutant $(\boldsymbol{C}, \boldsymbol{D})$. $\boldsymbol{E}$-I, Sections of E12.5 wild-type $(\boldsymbol{E}, \boldsymbol{F})$ and $\operatorname{Shh}-\boldsymbol{C}(\boldsymbol{G}, \boldsymbol{H})$ brains hybridized for Emx 1 show ventral ectopic expression in the mutant $(\boldsymbol{G}, \boldsymbol{H}$, red arrowheads). The plane of section is shown in $I . J, K, E m x 1$ expression on sections of E18.5 wild-type $(\boldsymbol{J})$ and $\operatorname{Sh} h-\mathrm{c}(\boldsymbol{K})$ brains. The black arrows indicate normal expression in hippocampus. The red arrowheads $(\boldsymbol{K})$ indicate neuroepithelial ectopic Emx1 expression in the mutant. $\boldsymbol{L}, \boldsymbol{M}, \mathrm{Tbr} 1$ expression on sections of E18.5 wild-type $(\boldsymbol{L})$ and $\operatorname{Sh} h$-c $(\boldsymbol{M})$ brains. The black arrows indicate normal expression in hippocampus. The black arrowhead in $L$ indicates EMT expression of $T b r 1$ in wild type. The red arrowhead in $M$ indicates one well defined layer of ectopic $T b r 1$ expression in the mutant. $\mathbf{N}, \mathbf{0}$, Foxg 1 expression labels the telencephalon $(\boldsymbol{N}, \mathbf{0})$ as well as the ectopic structure in the mutant $(\mathbf{O}$, arrow).

kinase-1 inhibitor able to block the Wnt pathway (Rena et al., 2004; Bryja et al., 2007). In explants cultured with D4476 the Foxb1 MAM expression domain increased in size (9 of 11) (Fig. $8 O-Q)$. These results suggested that the Wnt pathway, probably activated by Wnt8b, restricts the Foxb1 MAM expression domain in the neuroepithelium. In agreement, as Wnt $8 b$ expression increases in the MAM neuroepithelium during development, Foxb1 is downregulated in the neuroepithelium and upregulated in the newly born MBO neurons; by E12.5 the separation of the expression domains of both markers in the MAM was complete
(Fig. $8 R, S$ ). These results suggest that neural Shh maintains Foxb1 MBO expression, whereas Wnt8b inhibits it in the neuroepithelium, thereby restricting Foxb1 to neurons. Our results, together with some data from the literature (see Discussion), are summarized in Figure 8T.

\section{Discussion}

By analyzing a neuroepithelium-specific Shh mutant phenotype, we elucidate the differential roles of $S h h$ from the notochord and the neuroepithelium on hypothalamic development. We show 

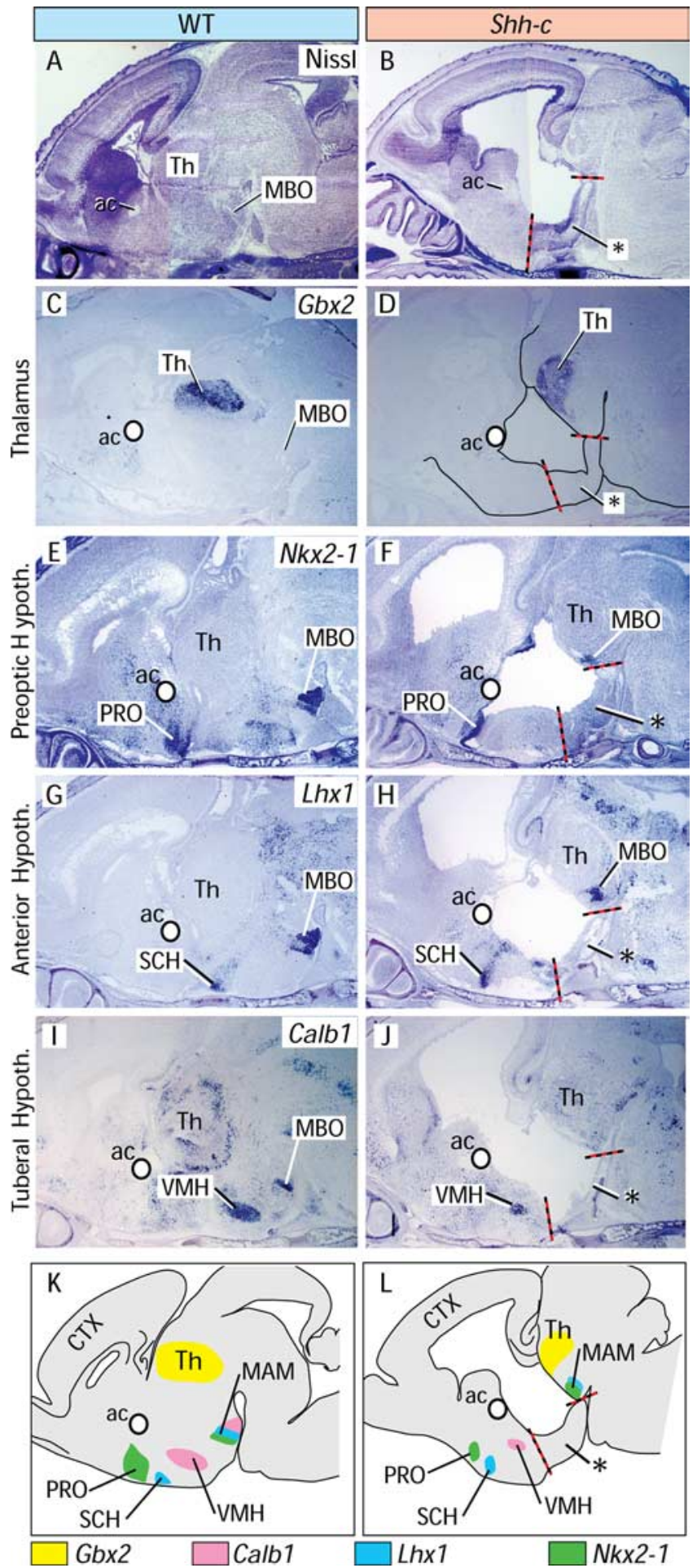

Figure 6. Neural Shh is required for differentiation in the medial hypothalamus. $\boldsymbol{A}, \boldsymbol{B}$, Nisslstained sagittal sections ofE18.5 wild-type $(\boldsymbol{A})$ and mutant $(\boldsymbol{B})$ brains. The red-black line indicates the boundaries of the abnormal layered structure (asterisk) in $\boldsymbol{B}$. $\boldsymbol{C}-\boldsymbol{J}$, ISH on sections of wild-type (C, $\boldsymbol{E}, \boldsymbol{G}$, $\boldsymbol{I})$ and $\operatorname{Shh}-\boldsymbol{C}(\boldsymbol{D}, \boldsymbol{F}, \boldsymbol{H}, \boldsymbol{J})$ E18.5 brains. A white circle marks the anterior commissure for reference. $\boldsymbol{C}, \boldsymbol{D}$, $G b \times 2$ labels the thalamus and was preserved in the mutant. $\boldsymbol{E}, \boldsymbol{F}$, Expression of $N k \times 2-1$ is preserved in the mutant PRO and MBO. G, $\boldsymbol{H}, L h \times 1$ is preserved in the mutant SCH and MBO. I, J, Calb1 is preserved in the mutant VMH but lost in the MBO. $K, \boldsymbol{L}$, Summary diagram of results.

that neural Shh is essential for the coordination of tissue growth and acquisition of AP and DV patterning in the hypothalamus and the DTJ. We demonstrate a role for neural Shh in growth and pattern coordination along the mediolateral axis of the hypothal- amus as well as in the specification of lateral hypothalamic neuronal subpopulations. We further show that neural Shh differentially promotes cell proliferation in the rostral and MAM regions. In addition, neural Shh influences the maintenance of Foxb1 expression in postmitotic MBO neurons by interplay with the Wnt pathway.

Finally, since our mutant expresses $N k \times 2-1$ in the ventral diencephalon and has two eyes, our results imply that neural Shh is dispensable for hypothalamic regional specification and eye field separation, two processes depending on Shh signaling (Chiang et al., 1996; Mathieu et al., 2002).

\section{Neural Shh in hypothalamic precursor expansion and neurogenesis}

Shh is needed early for proliferation in the diencephalon (Ishibashi and McMahon, 2002) and in particular in the caudal regions (Manning et al., 2006), which agrees with the reduced size of the hypothalamus in our mutant already at E9.5 (Fig. 3B). Our data indicate that this requirement is temporally restricted, and by E10.5 it has disappeared. However, we show that neural Shh has some promoting effect on proliferation in the rostral hypothalamus at E12.5, during the most active phase of hypothalamic neurogenesis (Altman and Bayer, 1986; Clancy et al., 2001). The effect of neural Shh on the neuroepithelium at this age could be direct, since weak expression of Gli1 and Ptch1 can be detected at ventral levels of the hypothalamic neuroepithelium through E12.5 and persists at least until E14.5 (data not shown). The effect of neural Shh could also be indirect, as in the early dorsal diencephalic proliferation (Ishibashi and McMahon, 2002).

Shh is necessary for cell viability in the diencephalic neuroepithelium at E9.0 (Ishibashi and McMahon, 2002) and in the midbrain-hindbrain junction until E10.5 (Blaess et al., 2006). We show, however, that in the hypothalamus this effect is temporally restricted, and it has disappeared by E10.5.

\section{Neural Shh and AP patterning in the hypothalamus}

In $S h h-c$ brains, reduced growth of the medial hypothalamus causes the hypothalamic mantle to become abnormally separated into a rostral subdivision (the three rostral hypothalamic regions) and a caudal one (the MAM). The gap that is created in this way unexpectedly confirms neural plate fate-mapping data in zebrafish showing that the presumptive MAM is indeed caudal to, but not continuous with the rest of the hypothalamus (Staudt and Houart, 2007). This region has been considered a somewhat atypical part of the hypothalamus from the point of view of specification mechanisms (Mathieu et al., 2002), patterning (Puelles and Rubenstein, 2003), connectivity (Thompson and Swanson, 2003), and function (Stackman and Taube, 1998; Vann and Aggleton, 2004; Radyushkin et al., 2005).

In addition, the mutant MAM is extremely reduced and becomes contiguous with the thalamic eminence, altering the DV pattern of the diencephalon (see below).

\section{Neural Shh in the PTh and EMT}

It has been well documented that deficiency in neural Shh from the ZLI leads to abolition of the PTh (see citations in Introduction). Therefore, our mutant lacks a PTh, as reflected by the loss of $D l \times 2$ (Fig. $3 E, F$ ) and Arx expression (supplemental Fig. 1F, G, available at www.jneurosci.org as supplemental material). However, $L h x 5$, a specific marker of the EMT, is preserved in our mutant, suggesting that lack of neural Shh in this region performs a genetic dissection between the two regions [Tbr1, another specific marker of the thalamic eminence, is however lost in the 

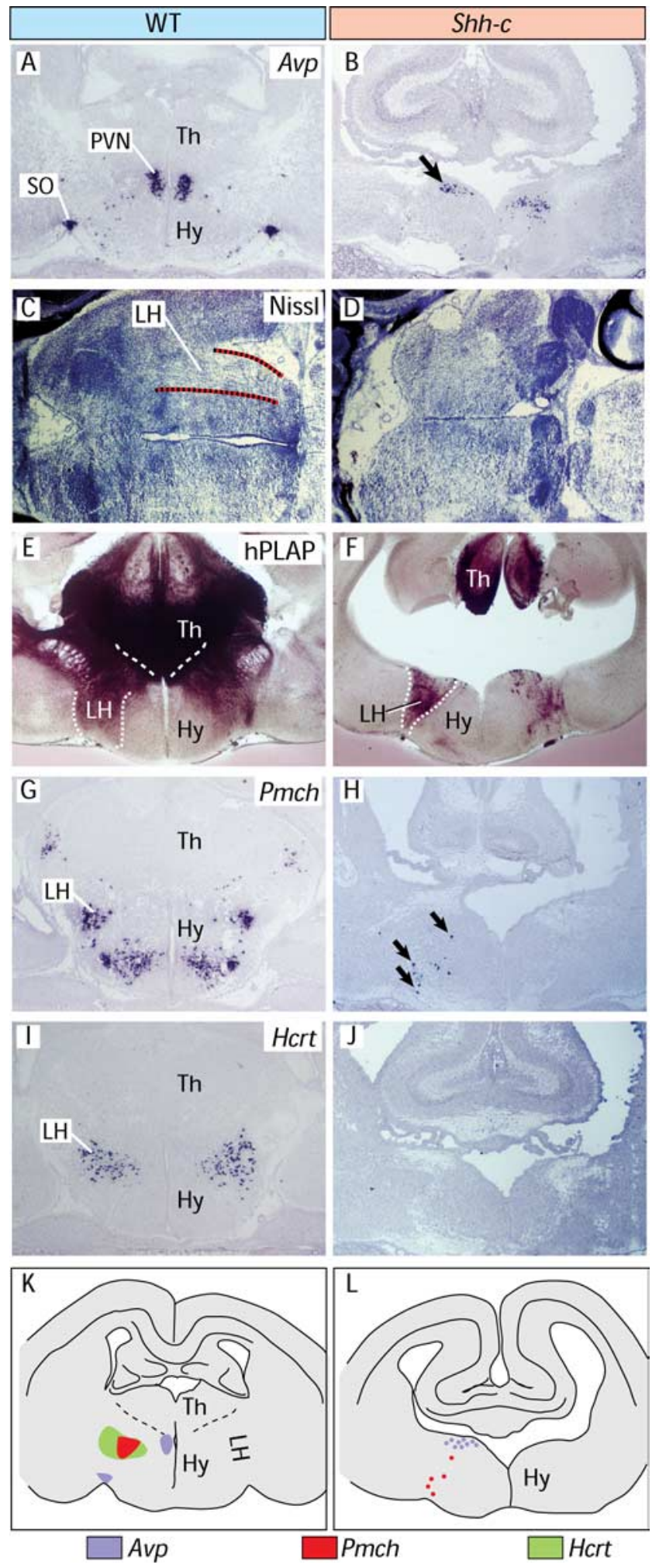

Figure 7. Neural Shh is required for lateral hypothalamus development. $A, B$, ISH on transverse sections of $E 18.5$ shows that the Avp expression domain is broader in the mutant and the S0 is absent. $\boldsymbol{C}, \boldsymbol{D}$, Horizontal sections through E18.5 wild-type $(\boldsymbol{C})$ and mutant $(\boldsymbol{D})$ brains. The lateral hypothalamus (delimited with dotted lines in $\boldsymbol{C}$ ) shows typically abundant longitudinal axons. A similar structure cannot be found in $\boldsymbol{D}$. $\boldsymbol{E}, \boldsymbol{F}, \mathrm{hPLAP}$ detection of the Foxb1 lineage in Foxb1-Cre/ZAP reporter mice (see Materials and Methods) shows the normal extent of the lateral hypothalamus $(\boldsymbol{E})$, very reduced in the Shh-c mutant $(\boldsymbol{F}) . \mathbf{G}, \boldsymbol{H}, P m c h$ neurons are very scarce in the mutant. $\boldsymbol{I}, \boldsymbol{J}, \mathrm{Hcr}$ neurons cannot be detected at all in the mutant. $\boldsymbol{K}, \boldsymbol{L}$, Summary diagrams of results. Expression is shown on the left side of each diagram, and abbreviations are on the right side. mutant (supplemental Fig. 1F, G, available at www.jneurosci.org as supplemental material)]. This reduces the size of the dorsal diencephalon and enlarges the communication between the lateral ventricles and the third ventricle.

\section{Neural Shh coordinates AP and DV patterning in the diencephalon and the formation of the DTJ}

Surprisingly, the patterning defects in dorsal and ventral diencephalon compound to alter the DTJ, which in the mutant extends ventrally to reach the midline (Fig. 3O,P). This is reminiscent of the major role played by neural Shh in the coordination of the AP and DV patterning in the midbrain-hindbrain junction (Blaess et al., 2006). In wild-type embryos, the growth of the thalamus and hypothalamus forces the expanding medial pallium to acquire a characteristic curved shape (compare, for instance, the hippocampus of wild type and mutant in Fig. $5 L, M$ ). In the Shh-c embryo, the very reduced hypothalamus (and thalamus) does not oppose resistance to the pallium, which stretches unimpeded toward the midline, particularly on the ventral side (Fig. 5A-D). At later stages, this fact has as an unexpected consequence the presence of part of the pallium (presumably the hippocampus) mispositioned at the caudal end of the ventral hypothalamus in the mutant.

\section{Neural Shh in mediolateral hypothalamic patterning}

Mediolateral patterning of the hypothalamus involves the specification of its distinctive medial and lateral zones. Between E9.5 and E13.0 approximately, Shh is expressed as a longitudinal band in the basal plate of the ventral hypothalamus (Shimamura et al., 1995). This expression domain coincides in time (E10.3-E12.7) (Altman and Bayer, 1986) and space (Altman and Bayer, 1986; Marchand et al., 1986), with the primordium of the lateral hypothalamus. We show that abolition of Shh expression in the hypothalamic neuroepithelium results in a very reduced lateral hypothalamus in which some of the most functionally important and characteristic neuronal subpopulations are either very reduced (Pmch neurons) or completely missing (Hcrt/orexin neurons). This indicates that neural Shh has a very important and specific role in the development of the lateral hypothalamus, possibly mediated by regulation of $D l \times 2, D b x 1$, and Foxd1 (Fig. 3).

\section{An interplay between $S h h$ and $W n t 8 b$ regulates expression of Foxb1, a crucial regulator of caudal hypothalamus development}

Deficiency in neural Shh translates also in incomplete differentiation of hypothalamic nuclei. We used the MBO as model to analyze this defect, since in the mutant this nucleus expresses several specific differentiation markers (Nkx2-1, Lhx1), but fails to express others like Sim1, Otp, and Foxb1. The case of Foxb1 is particularly intriguing, since it is expressed by the neural plate (E8.5) in the Shh full mutant (Fig. 1 $I, J$ ) but fails to be expressed at E18.5 in the Shh-c MBO (Fig. 8A,B). Our explant experiments show that, at E12.5, the dependence on Shh has disappeared. Since Foxb1 is still missing in the mutant MBO at E18.5, we conclude that neural Shh is necessary for maintenance of Foxb1 expression.

Incidentally, since Foxb1 is essential for the survival of the MBO neurons beyond the day of birth (Alvarez-Bolado et al., 2000), this result reveals an unexpected requirement for neural Shh in differentiation and survival of the MAM and is in keeping with data showing a role of Shh in neuronal survival in other systems (Miao et al., 1997; Oppenheim et al., 1999).

We also show that Wnt signaling suppresses Foxb1 expression 

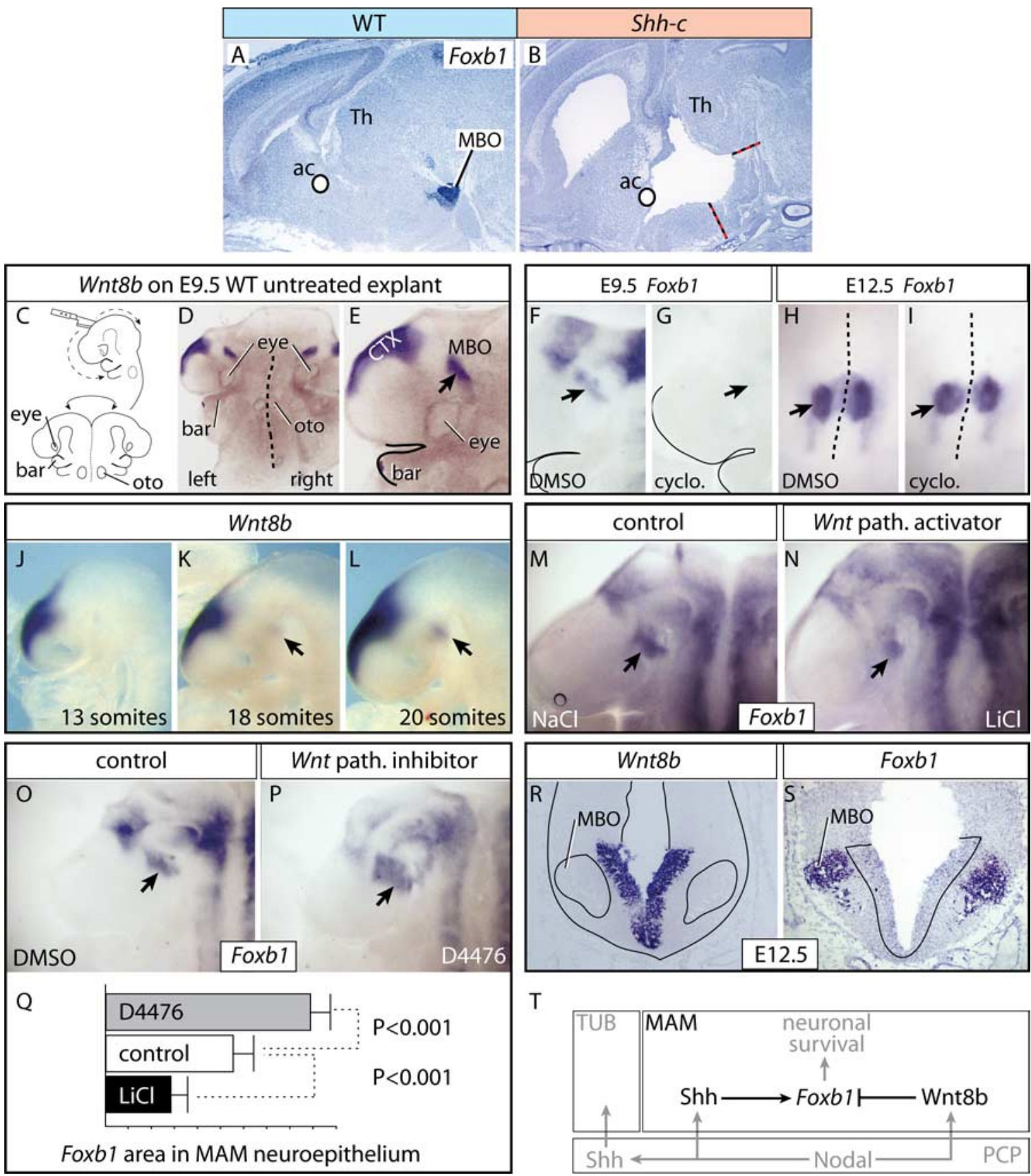

Figure 8. Neural Shh and Wnt pathway maintain and restrict expression of MAM survival factor Foxb1. $\boldsymbol{A}, \boldsymbol{B}$, Foxb1 expression on sagittal sections of wild-type $(\boldsymbol{A})$ and Shh-c $(\boldsymbol{B})$ E18.5 brains. The specific expression in the MBO $(\boldsymbol{A})$ is not present in the mutant $(\boldsymbol{B})$. C, Diagrams showing forebrain explant preparation. $\boldsymbol{D}, \boldsymbol{E}$, Appearance of the E9.5 mouse brain explants at lower $(\boldsymbol{D})$ and higher (E) magnification. This explant is labeled for Wnt8b. $\boldsymbol{F}$ - I, Treatment with Shh pathway blocker cyclopamine at E9.5 eliminates Foxb1 expression in the forebrain including MAM (arrows; compare $\boldsymbol{F}$, G). At E12.5, there is no effect on the MBO (arrows; compare $\boldsymbol{H}, \boldsymbol{I})$. $\boldsymbol{J}-\boldsymbol{L}$, Development of $W n t 8 b$ expression in the MAM. Expression is absent at 13 somites $(\boldsymbol{J})$, weakly detectable at 18 somites $(\boldsymbol{K}$, arrow), and fully developed at 20 somites ( $\boldsymbol{L}$, arrow). $\boldsymbol{M}, \boldsymbol{N}$, Treatment with Wnt pathway (path.) activator LiCl reduces the Foxb1 expression domain in the MAM neuroepithelium (arrow). $\mathbf{O}, \boldsymbol{P}$ Treatment with Wnt pathway inhibitor D4476 increases the domain of Foxb1 expression in the MAM neuroepithelium (arrow). $\mathbf{Q}$, The Foxb1-expressing MAM neuroepithelial surface area (in arbitrary units) was significantly larger after treatment with D4476 and significantly smaller after treatment with LiCl. Control explants cultured with DMSO. Shown are mean \pm SD. $R$, S, As Wnt8b expression $(\boldsymbol{R})$ increases in the MAM neuroepithelium, Foxb1 (S) decreases and becomes restricted to the mantle layer. The section boundaries have been outlined in $\boldsymbol{R}$, and the Wnt8b domain in $\boldsymbol{S}$. $\boldsymbol{T}$, Diagram showing the control of Foxb1 expression in the MAM. In black is shown our data, and in gray, data from the literature.

in progenitors (neuroepithelium), and therefore it counterbalances or modulates the positive effect of Shh in Foxb1 expression maintenance. The Wnt ligand involved here could be Wnt $8 \mathrm{~b}$, a specific marker of the MAM in all vertebrates (Cui et al., 1995; Hollyday et al., 1995; Kelly et al., 1995; Lako et al., 1998; Richardson et al., 1999; Garda et al., 2002; Houart et al., 2002). Wnt8b downregulates Foxb1 in the MAM of zebrafish (Kim et al., 2002), controls mammillary neurogenesis (Lee et al., 2006), and could be a local organizer (Erter et al., 2001; Houart et al., 2002). The interplay Shh/Wnt in Foxb1 control is presumably downstream the Nodal pathway (Strähle et al., 1996; Erter et al., 2001; Rohr et al., 2001; Houart et al., 2002; Mathieu et al., 2002) (Fig. 8T).

\section{References}

Acampora D, Postiglione MP, Avantaggiato V, Di Bonito M, Vaccarino FM, Michaud J, Simeone A (1999) Progressive impairment of developing 
neuroendocrine cell lineages in the hypothalamus of mice lacking the Orthopedia gene. Genes Dev 13:2787-2800.

Altman J, Bayer SA (1986) The development of the rat hypothalamus. Adv Anat Embryol Cell Biol 100:1-178.

Alvarez-Bolado G, Zhou X, Voss AK, Thomas T, Gruss P (2000) Winged helix transcription factor Foxb1 is essential for access of mammillothalamic axons to the thalamus. Development 127:1029-1038.

Arimatsu Y, Ishida M, Kaneko T, Ichinose S, Omori A (2003) Organization and development of corticocortical associative neurons expressing the orphan nuclear receptor Nurr1. J Comp Neurol 466:180-196.

Bachy I, Vernier P, Retaux S (2001) The LIM-homeodomain gene family in the developing Xenopus brain: conservation and divergences with the mouse related to the evolution of the forebrain. J Neurosci 21:7620-7629.

Blaess S, Corrales JD, Joyner AL (2006) Sonic hedgehog regulates Gli activator and repressor functions with spatial and temporal precision in the $\mathrm{mid} /$ hindbrain region. Development 133:1799-1809.

Blaess S, Stephen D, Joyner AL (2008) Gli3 coordinates three-dimensional patterning and growth of the tectum and cerebellum by integrating Shh and Fgf8 signaling. Development 135:2093-2103.

Bryja V, Schulte G, Arenas E (2007) Wnt-3a utilizes a novel low dose and rapid pathway that does not require casein kinase 1-mediated phosphorylation of Dvl to activate beta-catenin. Cell Signal 19:610-616.

Bulfone A, Smiga SM, Shimamura K, Peterson A, Puelles L, Rubenstein JL (1995) T-brain-1: a homolog of Brachyury whose expression defines molecularly distinct domains within the cerebral cortex. Neuron 15:63-78.

Burdakov D, Luckman SM, Verkhratsky A (2005) Glucose-sensing neurons of the hypothalamus. Philos Trans R Soc Lond B Biol Sci 360:2227-2235.

Caqueret A, Yang C, Duplan S, Boucher F, Michaud JL (2005) Looking for trouble: a search for developmental defects of the hypothalamus. Horm Res 64:222-230.

Chiang C, Litingtung Y, Lee E, Young KE, Corden JL, Westphal H, Beachy PA (1996) Cyclopia and defective axial patterning in mice lacking Sonic hedgehog gene function. Nature 383:407-413.

Clancy B, Darlington RB, Finlay BL (2001) Translating developmental time across mammalian species. Neuroscience 105:7-17.

Cui Y, Brown JD, Moon RT, Christian JL (1995) Xwnt-8b: a maternally expressed Xenopus Wnt gene with a potential role in establishing the dorsoventral axis. Development 121:2177-2186.

Dassule HR, Lewis P, Bei M, Maas R, McMahon AP (2000) Sonic hedgehog regulates growth and morphogenesis of the tooth. Development 127:4775-4785.

Dou C, Ye X, Stewart C, Lai E, Li SC (1997) TWH regulates the development of subsets of spinal cord neurons. Neuron 18:539-551.

Ericson J, Briscoe J, Rashbass P, van Heyningen V, Jessell TM (1997) Graded sonic hedgehog signaling and the specification of cell fate in the ventral neural tube. Cold Spring Harb Symp Quant Biol 62:451-466.

Erter CE, Wilm TP, Basler N, Wright CV, Solnica-Krezel L (2001) Wnt8 is required in lateral mesendodermal precursors for neural posteriorization in vivo. Development 128:3571-3583.

Fan CM, Porter JA, Chiang C, Chang DT, Beachy PA, Tessier-Lavigne M (1995) Long-range sclerotome induction by sonic hedgehog: direct role of the amino-terminal cleavage product and modulation by the cyclic AMP signaling pathway. Cell 81:457-465.

Garda AL, Puelles L, Rubenstein JL, Medina L (2002) Expression patterns of Wnt $8 \mathrm{~b}$ and Wnt7b in the chicken embryonic brain suggest a correlation with forebrain patterning centers and morphogenesis. Neuroscience 113:689-698.

Guinazu MF, Chambers D, Lumsden A, Kiecker C (2007) Tissue interactions in the developing chick diencephalon. Neural Dev 2:25.

Gulisano M, Broccoli V, Pardini C, Boncinelli E (1996) Emx1 and Emx2 show different patterns of expression during proliferation and differentiation of the developing cerebral cortex in the mouse. Eur J Neurosci 8:1037-1050.

Gunhaga L, Jessell TM, Edlund T (2000) Sonic hedgehog signaling at gastrula stages specifies ventral telencephalic cells in the chick embryo. Development 127:3283-3293.

Hashimoto-Torii K, Motoyama J, Hui CC, Kuroiwa A, Nakafuku M, Shimamura K (2003) Differential activities of Sonic hedgehog mediated by Gli transcription factors define distinct neuronal subtypes in the dorsal thalamus. Mech Dev 120:1097-1111.

Hatini V, Tao W, Lai E (1994) Expression of winged helix genes, BF-1 and
BF-2, define adjacent domains within the developing forebrain and retina. J Neurobiol 25:1293-1309.

Herrera E, Marcus R, Li S, Williams SE, Erskine L, Lai E, Mason C (2004) Foxd1 is required for proper formation of the optic chiasm. Development 131:5727-5739.

Hirata T, Nakazawa M, Muraoka O, Nakayama R, Suda Y, Hibi M (2006) Zinc-finger genes Fez and Fez-like function in the establishment of diencephalon subdivisions. Development 133:3993-4004.

Hollyday M, McMahon JA, McMahon AP (1995) Wnt expression patterns in chick embryo nervous system. Mech Dev 52:9-25.

Houart C, Caneparo L, Heisenberg C, Barth K, Take-Uchi M, Wilson S (2002) Establishment of the telencephalon during gastrulation by local antagonism of Wnt signaling. Neuron 35:255-265.

Hynes M, Porter JA, Chiang C, Chang D, Tessier-Lavigne M, Beachy PA, Rosenthal A (1995) Induction of midbrain dopaminergic neurons by Sonic hedgehog. Neuron 15:35-44.

Ingham PW, McMahon AP (2001) Hedgehog signaling in animal development: paradigms and principles. Genes Dev 15:3059-3087.

Ishibashi M, McMahon AP (2002) A sonic hedgehog-dependent signaling relay regulates growth of diencephalic and mesencephalic primordia in the early mouse embryo. Development 129:4807-4819.

Kelly GM, Greenstein P, Erezyilmaz DF, Moon RT (1995) Zebrafish wnt8 and wnt8b share a common activity but are involved in distinct developmental pathways. Development 121:1787-1799.

Kiecker C, Lumsden A (2004) Hedgehog signaling from the ZLI regulates diencephalic regional identity. Nat Neurosci 7:1242-1249.

Kim SH, Shin J, Park HC, Yeo SY, Hong SK, Han S, Rhee M, Kim CH, Chitnis AB, Huh TL (2002) Specification of an anterior neuroectoderm patterning by Frizzled8a-mediated Wnt8b signalling during late gastrulation in zebrafish. Development 129:4443-4455.

Kimura S, Hara Y, Pineau T, Fernandez-Salguero P, Fox CH, Ward JM, Gonzalez FJ (1996) The T/ebp null mouse: thyroid-specific enhancerbinding protein is essential for the organogenesis of the thyroid, lung, ventral forebrain, and pituitary. Genes Dev 10:60-69.

Kitamura K, Miura H, Yanazawa M, Miyashita T, Kato K (1997) Expression patterns of Brx1 (Rieg gene), Sonic hedgehog, Nkx2.2, Dlx1 and Arx during zona limitans intrathalamica and embryonic ventral lateral geniculate nuclear formation. Mech Dev 67:83-96.

Klein PS, Melton DA (1996) A molecular mechanism for the effect of lithium on development. Proc Natl Acad Sci U S A 93:8455-8459.

Koenen M, Rüther U, Müller-Hill B (1982) Immunoenzymatic detection of expressed gene fragments cloned in the lac $\mathrm{Z}$ gene of E. coli. EMBO J $1: 509-512$.

Komada M, Saitsu H, Kinboshi M, Miura T, Shiota K, Ishibashi M (2008) Hedgehog signaling is involved in development of the neocortex. Development 135:2717-2727.

Labosky PA, Winnier GE, Jetton TL, Hargett L, Ryan AK, Rosenfeld MG, Parlow AF, Hogan BL (1997) The winged helix gene, Mf3, is required for normal development of the diencephalon and midbrain, postnatal growth and the milk-ejection reflex. Development 124:1263-1274.

Lai CJ, Ekker SC, Beachy PA, Moon RT (1995) Patterning of the neural ectoderm of Xenopus laevis by the amino-terminal product of hedgehog autoproteolytic cleavage. Development 121:2349-2360.

Lako M, Lindsay S, Bullen P, Wilson DI, Robson SC, Strachan T (1998) A novel mammalian wnt gene, WNT8B, shows brain-restricted expression in early development, with sharply delimited expression boundaries in the developing forebrain. Hum Mol Genet 7:813-822.

Lee JE, Wu SF, Goering LM, Dorsky RI (2006) Canonical Wnt signaling through Lef1 is required for hypothalamic neurogenesis. Development 133:4451-4461.

Lewis PM, Dunn MP, McMahon JA, Logan M, Martin JF, St-Jacques B, McMahon AP (2001) Cholesterol modification of sonic hedgehog is required for long-range signaling activity and effective modulation of signaling by Ptc1. Cell 105:599-612.

Lobe CG, Koop KE, Kreppner W, Lomeli H, Gertsenstein M, Nagy A (1999) $\mathrm{Z} / \mathrm{AP}$, a double reporter for cre-mediated recombination. Dev Biol 208:281-292.

López-Martínez A, Chang DT, Chiang C, Porter JA, Ros MA, Simandl BK, Beachy PA, Fallon JF (1995) Limb-patterning activity and restricted posterior localization of the amino-terminal product of Sonic hedgehog cleavage. Curr Biol 5:791-796.

Lu S, Bogarad LD, Murtha MT, Ruddle FH (1992) Expression pattern of a 
murine homeobox gene, Dbx, displays extreme spatial restriction in embryonic forebrain and spinal cord. Proc Natl Acad Sci USA 89:8053-8057.

Machold R, Hayashi S, Rutlin M, Muzumdar MD, Nery S, Corbin JG, GritliLinde A, Dellovade T, Porter JA, Rubin LL, Dudek H, McMahon AP, Fishell G (2003) Sonic hedgehog is required for progenitor cell maintenance in telencephalic stem cell niches. Neuron 39:937-950.

Mann RK, Beachy PA (2004) Novel lipid modifications of secreted protein signals. Annu Rev Biochem 73:891-923.

Manning L, Ohyama K, Saeger B, Hatano O, Wilson SA, Logan M, Placzek M (2006) Regional morphogenesis in the hypothalamus: a BMP-Tbx2 pathway coordinates fate and proliferation through Shh downregulation. Dev Cell 11:873-885.

Marchand R, Lajoie L, Blanchet C (1986) Histogenesis at the level of the basal forebrain: the entopeduncular nucleus. Neuroscience 17:591-607.

Martí E, Bumcrot DA, Takada R, McMahon AP (1995) Requirement of 19K form of Sonic hedgehog for induction of distinct ventral cell types in CNS explants. Nature 375:322-325.

Mathieu J, Barth A, Rosa FM, Wilson SW, Peyriéras N (2002) Distinct and cooperative roles for Nodal and Hedgehog signals during hypothalamic development. Development 129:3055-3065.

Miao N, Wang M, Ott JA, D'Alessandro JS, Woolf TM, Bumcrot DA, Mahanthappa NK, Pang K (1997) Sonic hedgehog promotes the survival of specific CNS neuron populations and protects these cells from toxic insult in vitro. J Neurosci 17:5891-5899.

Michaud JL (2001) The developmental program of the hypothalamus and its disorders. Clin Genet 60:255-263.

Michaud JL, Rosenquist T, May NR, Fan CM (1998) Development of neuroendocrine lineages requires the bHLH-PAS transcription factor SIM1. Genes Dev 12:3264-3275.

Miyashita-Lin EM, Hevner R, Wassarman KM, Martinez S, Rubenstein JL (1999) Early neocortical regionalization in the absence of thalamic innervation. Science 285:906-909.

Oppenheim RW, Homma S, Marti E, Prevette D, Wang S, Yaginuma H, McMahon AP (1999) Modulation of early but not later stages of programmed cell death in embryonic avian spinal cord by sonic hedgehog. Mol Cell Neurosci 13:348-361.

Petryniak MA, Potter GB, Rowitch DH, Rubenstein JL (2007) Dlx1 and Dlx2 control neuronal versus oligodendroglial cell fate acquisition in the developing forebrain. Neuron 55:417-433.

Pierani A, Moran-Rivard L, Sunshine MJ, Littman DR, Goulding M, Jessell TM (2001) Control of interneuron fate in the developing spinal cord by the progenitor homeodomain protein Dbxl. Neuron 29:367-384.

Price M, Lazzaro D, Pohl T, Mattei MG, Rüther U, Olivo JC, Duboule D, Di Lauro R (1992) Regional expression of the homeobox gene Nkx-2.2 in the developing mammalian forebrain. Neuron 8:241-255.

Puelles L, Rubenstein JL (2003) Forebrain gene expression domains and the evolving prosomeric model. Trends Neurosci 26:469-476.

Puelles L, Kuwana E, Puelles E, Bulfone A, Shimamura K, Keleher J, Smiga S, Rubenstein JL (2000) Pallial and subpallial derivatives in the embryonic chick and mouse telencephalon, traced by the expression of the genes Dlx-2, Emx-1, Nkx-2.1, Pax-6, and Tbr-1. J Comp Neurol 424:409-438.

Puelles L, Martinez S, Martinez-de-la-Torre M, Rubenstein JLR (2004) Gene maps and related histogenetic domains in the forebrain and midbrain. In: The rat nervous system (Paxinos G, ed), pp 3-25. Amsterdam: Elsevier.

Radyushkin K, Anokhin K, Meyer BI, Jiang Q, Alvarez-Bolado G, Gruss P (2005) Genetic ablation of the mammillary bodies in the Foxb1 mutant mouse leads to selective deficit of spatial working memory. Eur J Neurosci 21:219-229.

Rena G, Bain J, Elliott M, Cohen P (2004) D4476, a cell-permeant inhibitor of CK1, suppresses the site-specific phosphorylation and nuclear exclusion of FOXO1a. EMBO Rep 5:60-65.

Richardson M, Redmond D, Watson CJ, Mason JO (1999) Mouse Wnt8B is expressed in the developing forebrain and maps to chromosome 19. Mamm Genome 10:923-925.

Roelink H, Porter JA, Chiang C, Tanabe Y, Chang DT, Beachy PA, Jessell TM (1995) Floor plate and motor neuron induction by different concentra- tions of the amino-terminal cleavage product of sonic hedgehog autoproteolysis. Cell 81:445-455.

Rohr KB, Barth KA, Varga ZM, Wilson SW (2001) The nodal pathway acts upstream of hedgehog signaling to specify ventral telencephalic identity. Neuron 29:341-351.

Scholpp S, Wolf O, Brand M, Lumsden A (2006) Hedgehog signalling from the zona limitans intrathalamica orchestrates patterning of the zebrafish diencephalon. Development 133:855-864.

Schwenk F, Baron U, Rajewsky K (1995) A cre-transgenic mouse strain for the ubiquitous deletion of loxP-flanked gene segments including deletion in germ cells. Nucleic Acids Res 23:5080-5081.

Shimamura K, Hartigan DJ, Martinez S, Puelles L, Rubenstein JL (1995) Longitudinal organization of the anterior neural plate and neural tube. Development 121:3923-3933.

Shoji H, Ito T, Wakamatsu Y, Hayasaka N, Ohsaki K, Oyanagi M, Kominami R, Kondoh H, Takahashi N (1996) Regionalized expression of the Dbx family homeobox genes in the embryonic CNS of the mouse. Mech Dev 56:25-39.

Simerly RB (2004) Anatomical substrates of hypothalamic integration. In: The rat nervous system, Ed 3 (Paxinos G, ed), pp 335-368. Amsterdam: Elsevier.

Skidmore JM, Cramer JD, Martin JF, Martin DM (2007) Cre fate mapping reveals lineage specific defects in neuronal migration with loss of Pitx2 function in the developing mouse hypothalamus and subthalamic nucleus. Mol Cell Neurosci 37:696-707.

Soriano P (1999) Generalized lacZ expression with the ROSA26 Cre reporter strain. Nat Genet 21:70-71.

Stackman RW, Taube JS (1998) Firing properties of rat lateral mammillary single units: head direction, head pitch, and angular head velocity. J Neurosci 18:9020-9037.

Stambolic V, Ruel L, Woodgett JR (1996) Lithium inhibits glycogen synthase kinase-3 activity and mimics wingless signalling in intact cells. Curr Biol 6:1664-1668.

Staudt N, Houart C (2007) The prethalamus is established during gastrulation and influences diencephalic regionalization. PLoS Biol 5:e69.

Stoykova A, Fritsch R, Walther C, Gruss P (1996) Forebrain patterning defects in Small eye mutant mice. Development 122:3453-3465.

Strähle U, Blader P, Ingham PW (1996) Expression of axial and sonic hedgehog in wildtype and midline defective zebrafish embryos. Int J Dev Biol 40:929-940.

Szabó NE, Zhao T, Zhou X, Alvarez-Bolado G (2009) The role of sonic hedgehog of neural origin in thalamic differentiation in the mouse. J Neurosci 29:2453-2466.

Takahashi T, Nowakowski RS, Caviness VS Jr (1993) Cell cycle parameters and patterns of nuclear movement in the neocortical proliferative zone of the fetal mouse. J Neurosci 13:820-833.

Thompson RH, Swanson LW (2003) Structural characterization of a hypothalamic visceromotor pattern generator network. Brain Res Brain Res Rev 41:153-202.

Vann SD, Aggleton JP (2004) The mammillary bodies: two memory systems in one? Nat Rev Neurosci 5:35-44.

Vieira C, Garda AL, Shimamura K, Martinez S (2005) Thalamic development induced by Shh in the chick embryo. Dev Biol 284:351-363.

Warren N, Caric D, Pratt T, Clausen JA, Asavaritikrai P, Mason JO, Hill RE, Price DJ (1999) The transcription factor, Pax6, is required for cell proliferation and differentiation in the developing cerebral cortex. Cereb Cortex 9:627-635.

Xuan S, Baptista CA, Balas G, Tao W, Soares VC, Lai E (1995) Winged helix transcription factor BF-1 is essential for the development of the cerebral hemispheres. Neuron 14:1141-1152.

Zeltser LM (2005) Shh-dependent formation of the ZLI is opposed by signals from the dorsal diencephalon. Development 132:2023-2033.

Zhao T, Zhou X, Szabó N, Leitges M, Alvarez-Bolado G (2007) Foxb1driven Cre expression in somites and the neuroepithelium of diencephalon, brainstem, and spinal cord. Genesis 45:781-787.

Zhao T, Szabó N, Ma J, Luo L, Zhou X, Alvarez-Bolado G (2008) Genetic mapping of Foxb1-cell lineage shows migration from caudal diencephalon to telencephalon and lateral hypothalamus. Eur J Neurosci 28:19411955. 